\title{
Unified View of Magnetic Nanoparticle Separation under Magnetophoresis
}

\author{
Sim Siong Leong, Zainal Ahmad, Siew Chun Low, Juan Camacho, Jordi Faraudo,* and JitKang Lim*
}

Cite This: Langmuir 2020, 36, 8033-8055

Read Online

ABSTRACT: The migration process of magnetic nanoparticles and colloids in solution under the influence of magnetic field gradients, which is also known as magnetophoresis, is an essential step in the separation technology used in various biomedical and engineering applications. Many works have demonstrated that in specific situations, separation can be performed easily with the weak magnetic field gradients created by permanent magnets, a process known as low-gradient magnetic separation (LGMS). Due to the level of complexity involved, it is not possible to understand the observed kinetics of LGMS within the classical view of magnetophoresis. Our experimental and theoretical investigations in the last years unravelled the existence of two novel physical

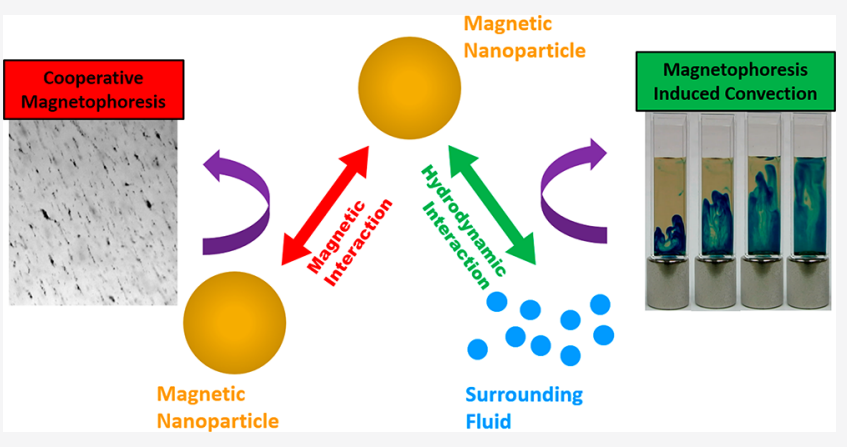
effects that speed up the magnetophoresis kinetics and explain the observed feasibility of LGMS. Those two effects are (i) cooperative magnetophoresis (due to the cooperative motion of strongly interacting particles) and (ii) magnetophoresis-induced convection (fluid dynamics instability originating from inhomogeneous magnetic gradients). In this feature article, we present a unified view of magnetophoresis based on the extensive research done on these effects. We present the physical basis of each effect and also propose a classification of magnetophoresis into four distinct regimes. This classification is based on the range of values of two dimensionless quantities, namely, aggregation parameter $N^{*}$ and magnetic Grashof number $G r_{\mathrm{m}}$, which include all of the dependency of LGMS on various physical parameters (such as particle properties, thermodynamic parameters, fluid properties, and magnetic field properties). This analysis provides a holistic view of the classification of transport mechanisms in LGMS, which could be particularly useful in the design of magnetic separators for engineering applications.

\section{INTRODUCTION}

Magnetophoresis is defined as the motion of magnetic particles, relative to their surrounding fluid, under the influence of an externally imposed magnetic field gradient. ${ }^{1}$ Until recently, magnetophoresis has received much less attention than other somewhat similar processes such as electrophoresis and dielectrophoresis (in which a charged or dipolar colloid migrates under suitable electric fields) which indeed have a long and established tradition in colloid and interface science. But the interest in magnetophoresis within the scientific community has increased in recent years, mainly due to the continuous development of novel magnetic particles designed for many applications, $^{2-11}$ which include drug delivery, the removal of heavy ions from water, the removal of microalgae, the sorting as well as detection of cells, and many more. The use of functionalized magnetic particles in these applications (instead of nonmagnetic particles) is justified by the possibility to remotely control their migration toward desired targets and/or to separate them from solution by using magnetic fields. Therefore, the use of these novel particles in actual applications depends critically on the magnetophoretic motion of particles which allows extraction or separation from the fluid. ${ }^{12}$ The feasibility of this magnetophoretic migration process can be easily illustrated with an experiment such as the example shown in Figure 1a. ${ }^{13}$ In this case, a magnet was placed adjacent to a bottle containing a suspension of magnetic particles to induce their migration toward the magnet. Eventually, all of the particles end up accumulated at the wall in contact with the magnet (and therefore separated from the suspension) in a few minutes. In Figure $1 \mathrm{~b}$, we show another similar experiment, but in this case, there are several magnets located around the bottle containing the dispersion. All particles were immobilized on the inner walls of the bottle in a few minutes (for a full video of the process, see ref 14), and the supernatant can be easily removed with a syringe. It is important to recall that all of these magnetic separation experiments were performed with diluted dispersions of magnetic particles. The previously mentioned magnetic separation processes cannot be applied to fluids containing

Received: March 25, 2020

Revised: June 12, 2020

Published: June 18, 2020 
(a)

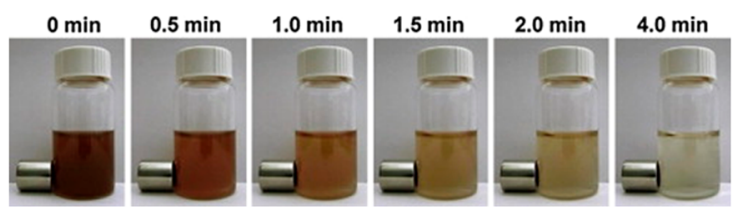

(c)

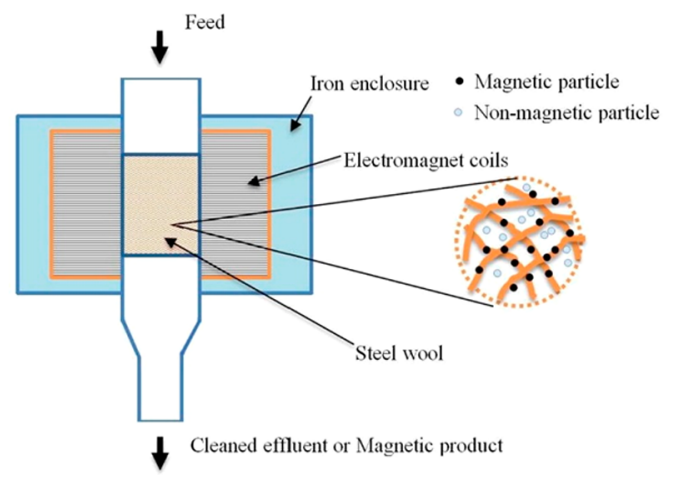

(b)

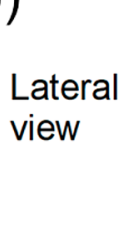

$\mathrm{t}=0$

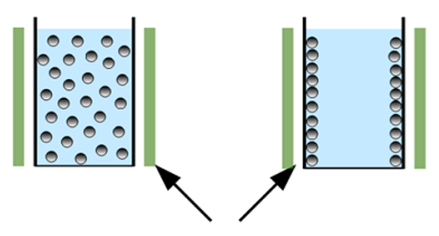

Top view
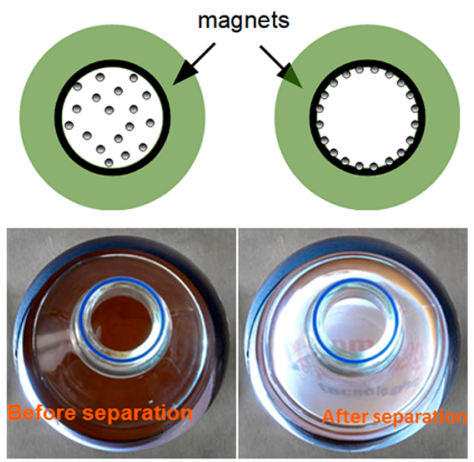

Figure 1. (a) Magnetophoretic collection of iron oxide particles of $\sim 150 \mathrm{~nm}$ hydrodynamic radius in real time by a grade N50 NdFeB magnet. (The magnetic gradient near the magnet is $\sim 50 \mathrm{~T} / \mathrm{m}$ and decays with distance from the magnet.) Reproduced with permission from ref 13 . Copyright 2014 Elsevier. (b) Scheme (lateral and top views) and actual picture (top view) of a cylindrical magnetophoretic separator made with permanent magnets which generate an approximately uniform radial gradient. The picture shows an actual cylindrical separator and a bottle inside containing a suspension of magnetic colloids before and after the magnetic separation (picture courtesy of Sepmag Systems SL). (c) Scheme of a high-gradient magnetic separator based on a separation column containing iron wool inside able to trap magnetic particles. The iron wool generates a high magnetic gradient in response to a uniform magnetic field generated by external coils. Reproduced with permission from ref 27. Copyright 2017 Elsevier.

extremely large concentrations of magnetic particles such as ferrofluids or magnetorheological fluids. ${ }^{15}$ In these magnetic fluids, magnetic particles exhibit collective behavior which includes a variety of complex phenomena (Rosensweig instability in ferrofluids, the magnetorheological effect, etc. $)^{16}$ that are beyond the scope of this feature article.

Magnetic separation has many advantages over traditional fixed-bed separation methods, such as activated carbon adsorption and affinity chromatography. In particular, functionalized magnetic particles offer a large exposed solid/fluid interface that serves as a magnetically controllable platform for the selective adsorption of many different entities in dispersion ${ }^{11}$ without the use of porous materials, which are often plagued by high mass-transfer resistances. ${ }^{17}$ Therefore, it is not surprising that magnetic separation has been presented as an alternative to typical centrifugation and filtration steps in industrial processes as well as in laboratory applications.

Substantial efforts have been directed toward the design of the improved magnetic particles, in particular, key aspects such as the design of their surface functionalization, ${ }^{18}$ the improvement of their magnetic response, ${ }^{19,20}$ and improved colloidal stability. ${ }^{21-26}$ However, in order to fully exploit such unique and attractive features exhibited by magnetic particles in real time applications, particle design alone is insufficient. It is also essential to establish system design rules to allow practical and efficient ways to recollect the particles from their suspension by using an externally applied magnetic field within a reasonable time scale.

Traditionally, magnetophoretic separation has been performed by using the so-called high-gradient magnetic separation (HGMS) technique. ${ }^{17}$ In an HGMS device, the dispersion containing the magnetic particles is pumped through a column filled with a packed bed of magnetizable material such as steel wool (stainless steel fibers on the order of a few micrometers), as shown in Figure 1c. An externally applied magnetic field magnetizes these fibers, which induce localized and high magnetic field gradients (typically $10^{2}-10^{4} \mathrm{~T} / \mathrm{m}$ ), ${ }^{27}$ trapping the particles within the packed fibers. This technique has been successfully applied to capture particles with sizes as small as 10 $\mathrm{nm}$, and it has found many uses over the years. ${ }^{12,17,27-32}$ However, in many applications, particularly in the biomedical field, the preferred strategy is the employment of permanent magnets which are located outside the particle suspension for distal control of the particle motion in noncontact mode, ${ }^{6,33}$ as shown in Figure 1a,b. As illustrated in Figure 1, it is possible to develop magnetophoretic separation devices with simple arrangements of permanent magnets by placing the dispersion inside the magnetic gradient without the need to direct the dispersion into a separation column. However, the use of permanent magnets in these magnetic separators limits the feasible magnetic gradient (which is the driving force for the motion of the magnetic particles) to below $100 \mathrm{~T} / \mathrm{m}$, which is much smaller than the field gradients employed in HGMS. In contrast to HGMS, we named this process low-gradient magnetic separation (LGMS) ${ }^{34,35}$ for situations such as those shown in Figure 1a,b in which the gradient arises from external permanent magnets. In spite of the apparent simplicity of the processes shown in Figure 1a,b, it must be noted that the real life implementation of LGMS is extremely challenging since the required motion on macroscopic time and length scales has to be induced by magnetic forces operating over magnetizable volumes at the nanoscale. ${ }^{36}$ Not many years ago, it was even argued that given the magnitude of the factors opposing magnetophoresis and the small driving forces, the fact that lowgradient magnetophoresis could be actually observed for nanosized particles was considered a paradox. ${ }^{37}$

The fact is that, under appropriate conditions, the process of LGMS can be actually observed and employed (as in Figure 
1a,b) whereas under other conditions the process is so slow that it is not useful for any practical purpose. The conditions under which LGMS is possible have been studied extensively, both from a fundamental point of view ${ }^{34,35,38-45}$ (elucidation of basic physical mechanisms) and also from an applied point of view. ${ }^{29,46-49}$ All of these studies have revealed complex and rich phenomena, occurring on both the colloidal and the macroscopic scales. For example, if the dipole-dipole interaction between superparamagnetic particles magnetized by the external field is strong enough, then a cooperative mechanism substantially speeds up the magnetophoretic motion, with separation kinetics strongly dependent on the concentration of particles. ${ }^{34,35,50,51}$ Also, recent works have demonstrated both experimentally and by computer simulations that under certain conditions (magnetic gradients strongly localized in a capture region) a macroscopic hydrodynamic instability appears, generating a convective flow, and such a scenario has substantially sped up the magnetophoretic process. ${ }^{52-54}$ The detailed analysis presented in these studies shows that the origin of the hydrodynamic instability is the inhomogeneity of the magnetophoretic force, which subsequently causes an inhomogeneous magnetophoretic drift and is counteracted by the induced convective flow. This mechanism is reminiscent of a hydrodynamic instability observed in microfluidic mixers when fluids with a mismatch in magnetizations (a ferrofluid and a nonmagnetic liquid, for example) interact with a uniform magnetic field in a microfluidic chamber. ${ }^{55}$ In both cases, the inhomogeneity in magnetic properties (magnetophoretic force in the first case, magnetization in the second case) induces a hydrodynamic instability, with useful practical consequences (macroscopic convection in the first case, microfluidic mixing in the second case).

Both cooperative and hydrodynamic effects will be discussed in detail later in this feature article. The important point to recall here is that the separation kinetics depends on several different physical mechanisms that collectively impart complex, rich behavior. The prediction of the occurrence of these effects in magnetophoresis and the quantitative prediction of its kinetic behavior remain challenging. This scenario is mainly due to the complex interplay between physical parameters that span many different system scales, including particle-level properties such as particle size ${ }^{40}$ magnetic content, and shape ${ }^{56}$ and macroscale properties such as particle concentration ${ }^{34,57}$ and the magnetic field gradient. ${ }^{52}$

The objective of this feature article is to combine the available theoretical and experimental results in order to provide a unified theoretical framework for the description of the separation mechanism of low-gradient magnetophoresis in the form of dimensionless numbers that characterize the different regimes of the magnetophoresis process. In the spirit of classical fluid dynamics, our aim here is to establish the criteria (based on dimensionless analysis) to characterize the flow behavior of magnetic particles during magnetophoresis, more specifically, under the influences of (i) particle self-assembly induced by the magnetic field (cooperative magnetophoresis) and (ii) magnetophoresis-induced convection. Our focus in this feature article is to provide a unified view of how these physical phenomena define the dynamical behavior of magnetophoresis. Also, we provide a classification, based on dimensionless numbers, to identify the mechanisms which govern the kinetics of the magnetic separation process under different operating conditions, including the concentration, magnetic field gradient, and particle size and magnetization.
The feature article is organized as follows. We first describe basic concepts of magnetic particles and magnetophoresis, including an outline of the basic transport mechanisms of colloids under magnetic field gradients. Then the dimensionless numbers that characterize the importance of these mechanisms are introduced. Later, by using these dimensionless numbers, we describe the separation mechanisms of low-gradient magnetophoresis under different operating conditions. In addition, we also discuss some of the engineering applications which can be benefitted by the understanding of the LGMS process. Finally, we summarize the main findings related to the LGMS process in the Conclusions.

\section{FUNDAMENTAL CONCEPTS: SUPERPARAMAGNETIC PARTICLES AND THEIR TRANSPORT BEHAVIOR UNDER MAGNETIC FIELD GRADIENTS}

Superparamagnetic Particles. Before considering the magnetophoresis process itself, it is important to understand the physical properties (particularly the magnetic response) of the magnetic particles to be used in the magnetophoresis experiments. The magnetic nanoparticles (MNPs) usually employed in LGMS are superparamagnetic. Superparamagnetism is a peculiar magnetic behavior found in small crystals of magnetic materials that are a few nanometers in size, such as iron oxide. It is also possible to obtain larger superparamagnetic particles $(100-1000 \mathrm{~nm})$ as composite colloids made of a large number of superparamagnetic nanoparticles inside the matrix of a nonmagnetic material such as silica or latex. Superparamagnetism is characterized by the fact that when a magnetic field is applied to a superparamagnetic particle, the magnetization can be very large (as in ferromagnetic materials), but when the field is zero, the magnetization disappears. ${ }^{58-60}$ This highly tunable behavior makes these materials attractive for applications in combination with magnetophoresis since superparamagnetism makes it possible to switch the magnetization of these particles on or off by using external magnetic fields, which subsequently enables the control of their colloidal behavior and motion.

The origin of superparamagnetism can be qualitatively understood by considering the size effect in the magnetic response of a crystal, as illustrated in Figure 2a. Macroscopic crystals of materials that exhibit ferromagnetic or ferrimagnetic behavior contain domains with well-defined size and magnetization, which are separated by domain walls. Under the effect of a magnetic field, the magnetization of such a multidomain sample changes by displacements of the domain walls. These domains have a characteristic minimum size $D_{\mathrm{cr}}$, which is specific for each material. Crystals smaller than this size (typically below $100 \mathrm{~nm}$ ) contain only a single magnetic domain; ${ }^{58,59}$ that is, they are in a state of uniform magnetization even in the absence of any external magnetic field (Figure 2a). For a single-domain particle, domain wall displacement is not possible (since there are no domain walls), and a change in magnetization by an external field requires the rotation of the magnetic moment of the entire particle. For the larger particles, this is inhibited by the large anisotropy energy barrier, but for sufficiently small nanocrystals, the monodomain magnetization inside the particle is energetically unstable and the magnetic dipole of the particle experiences continuous thermal flipping (Figure 2a). This particular situation is called the superparamagnetic state. ${ }^{60}$ In the superparamagnetic state, the spontaneous flipping of the magnetic moment of the nanocrystal is so fast that in the absence 
(a) Multidomain Monodomain Superparamagnetism

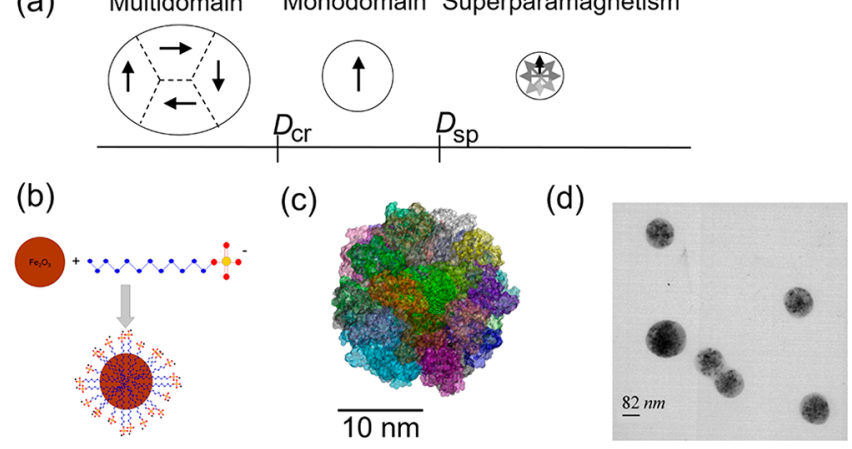

Figure 2. (a) Scheme indicating the different behavior of magnetic particles depending on their size. $D_{\text {cr }}$ indicates the critical size for monodomain behavior, and $D_{s p}$ indicates the critical size for superparamagnetic behavior. (b) Scheme of a typical preparation of magnetic nanoparticles covered by surfactants, such as those employed in ref 40 in which $10 \mathrm{~nm} \gamma$ - $\mathrm{Fe}_{2} \mathrm{O}_{3}$ nanocrystals were stabilized by the adsorption of anionic SDS surfactant. (c) Snapshot from computer simulations reproducing with full atomistic detail the preformed protein corona ( $\sim 6 \mathrm{~nm}$ thickness) of human serum albumin protein designed to fully protect a $9 \mathrm{~nm}$ iron oxide nanoparticle. (See full details of the simulations and experiments in ref 64.) Reproduced with permission from ref 64. Copyright 2019 American Chemical Society. (d) Image of superparamagnetic colloids made by a polystyrene matrix with iron oxide nanoparticles inside (dark spots). Reproduced with permission from Martinez Pedrero. ${ }^{67}$

of any applied field the measured magnetic moment is zero. In the presence of an applied field, the particles stabilize their dipole in the direction of the field and behave like a paramagnetic material but with a large saturation magnetization (corresponding to that of a single domain), hence the name "superparamagnetic" state. The critical size for superparamagnetism $D_{\text {sp }}$ is typically 1 order of magnitude smaller than the critical domain size, $D_{\mathrm{cr}}$, and normally on the order of $D_{\mathrm{sp}} \approx 10$ nm. ${ }^{61}$

In the absence of any specific treatment, magnetic nanocrystals are not soluble in water. Small suspended solids in solution have a tendency to decrease their surface energy by aggregation, reducing in this way the solid/fluid interface. In order to obtain stable dispersions of superparamagnetic nanoparticles, ${ }^{21}$ a typical strategy is to modify and/or functionalize the surface of the nanocrystals ${ }^{18}$ in order to prevent the agglomeration of MNPs by imposing a physical barrier or electric charges onto the surface of MNPs. ${ }^{23}$ For instance, colloidally stable and uniform MNPs can be obtained by adding surfactant to the reactive media during the synthesis process, ${ }^{62}$ as shown in Figure $2 \mathrm{~b}$. Additionally, the sintered MNP powders can also be redispersed back into aqueous media after surface modification with polyelectrolyte so that the resulting MNP suspension maintains its colloidal stability for more than 6 months. ${ }^{26}$ Besides producing a colloidally stable MNP system, surface modification also provides specific functionality to MNPs. ${ }^{18}$ In the case of biological applications, MNPs can also be given a sort of biological identity by coating them with a preformed protein corona, ${ }^{63,64}$ as seen in Figure $2 \mathrm{c}$. In that way, the magnetic nanocrystal (usually made by metals or metal oxides) avoids direct interaction with the environment. This strategy has been employed in order to make iron oxide magnetic nanoparticles biocompatible and make them suitable as magnetic carriers for anticancer drugs ${ }^{65}$ and for magnetic resonance imaging applications. ${ }^{64}$ In all of these applications, MNPs are employed in concentrations that can be considered to be very dilute. For example, in the magnetic resonance experiments performed in ref 66 , the employed volume fractions were on the order of $10^{-6}-10^{-7}$ depending on the experiment. In the magnetophoretic studies in ref 53, the experiments employed concentrations of $10-100 \mathrm{mg} / \mathrm{L}$ of $30 \mathrm{~nm}$ PEG-coated MNPs, corresponding to volume fractions of only $3 \times 10^{-5}-3$ $\times 10^{-6}$. At higher concentrations (4\% in volume or larger), superparamagnetic nanocrystals can also be employed to prepare ferrofluids which have many applications as smart materials. ${ }^{15,16}$

Another option is to coat the nanoparticles with a nonmagnetic material such as silica in order to obtain a single-core magnetic particle. ${ }^{19}$ This approach can be extended in order to obtain much larger magnetic colloidal particles $(100 \mathrm{~nm}-2 \mu \mathrm{m})$ made of a nonmagnetic matrix (for example, polymer or silica) which contains a large number of superparamagnetic nanocrystals that are a few nanometers inside, ${ }^{68,69}$ as illustrated in Figure $2 \mathrm{~d}$. Provided that the magnetic nanocrystals are well separated inside the matrix (avoiding mutual interactions), the response of the resulting composite particle is still superparamagnetic, allowing magnetic dipoles much larger than those obtained from single nanocrystals. These composite colloids are sometimes denoted as magnetic beads by many commercial suppliers of particles for magnetophoretic separation. Another interesting property of these magnetic beads is that they are fabricated with a density close to that of water in order to prevent sedimentation. (Typical densities seen in the catalogs of the manufacturers are $\sim 1.02 \mathrm{~g} / \mathrm{cm}^{3}$ for low-density beads and $1.2 \mathrm{~g} /$ $\mathrm{cm}^{3}$ for high-density beads.) These densities are possible because of the low density of the employed nonmagnetic matrix that compensates for the much higher density of the embedded magnetic nanocrystals.

It is worth emphasizing that superparamagnetic particles, in the form of either MNPs or magnetic beads, are particularly interesting due to their highly tunable behavior. In the absence of an applied magnetic field, they behave as nonmagnetic particles. In the presence of a uniform magnetic field, they acquire a magnetization $\overrightarrow{\mathbf{M}}=\overrightarrow{\mathbf{M}}(\overrightarrow{\mathbf{B}})$ with a magnitude that is linearly dependent on the modulus of applied field $|\overrightarrow{\mathbf{B}}|$ for small fields and saturates at a saturation magnetization $M_{\text {sat }}$ in larger fields, closely following a Langevin function (such as for paramagnetism $){ }^{40,60,70}$ It is important to recall here that the effect of a uniform magnetic field over a superparamagnetic particle is to magnetize it; however, in order to move a magnetic particle and to observe magnetophoresis, an inhomogeneous magnetic field is needed.

Theory of Magnetophoresis. The force experienced by a superparamagnetic nanocrystal of volume $V_{\mathrm{m}}$ due to an applied magnetic field gradient is given by ${ }^{40,71-73}$

$$
\overrightarrow{\mathbf{F}}_{\text {mag }}=|\overrightarrow{\mathbf{m}}(\overrightarrow{\mathbf{B}})| \vec{\nabla} B=V_{\mathrm{m}} \rho_{\mathrm{m}}|\overrightarrow{\mathbf{M}}(\overrightarrow{\mathbf{B}})| \vec{\nabla} B
$$

where $\vec{\nabla} B$ is the gradient of the modulus of the magnetic field $B$ and $|\overrightarrow{\mathbf{m}}(\overrightarrow{\mathbf{B}})|$ is the modulus of the magnetic dipole $\rightarrow$ of the nanocrystal as induced by the magnetic field strength $\overrightarrow{\mathbf{B}}$ at the position of the particle. It should be emphasized that eq 1 is not generally applicable to magnetic particles but is specific for superparamagnetic particles due to its magnetoquasistatic nature $(\nabla \times \vec{B}=0)$ and the spontaneous alignment of particle magnetization along the magnetic field $(\overrightarrow{\mathbf{M}} \| \overrightarrow{\mathbf{B}})) \cdot{ }^{72,73}$ In practice, the magnetic dipole can be computed from the magnetization per unit mass $|\overrightarrow{\mathbf{M}}(\overrightarrow{\mathbf{B}})|$ obtained in a typical magnetic character- 


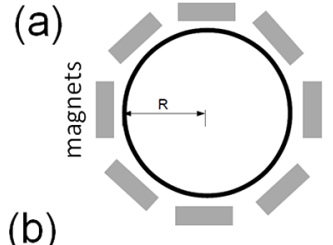

(b)

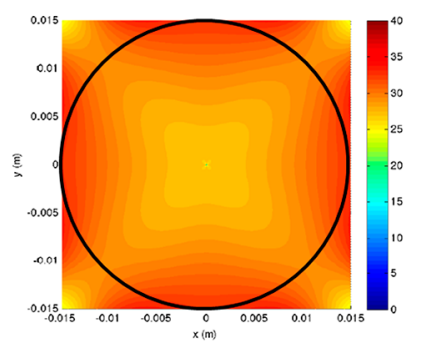

(c)

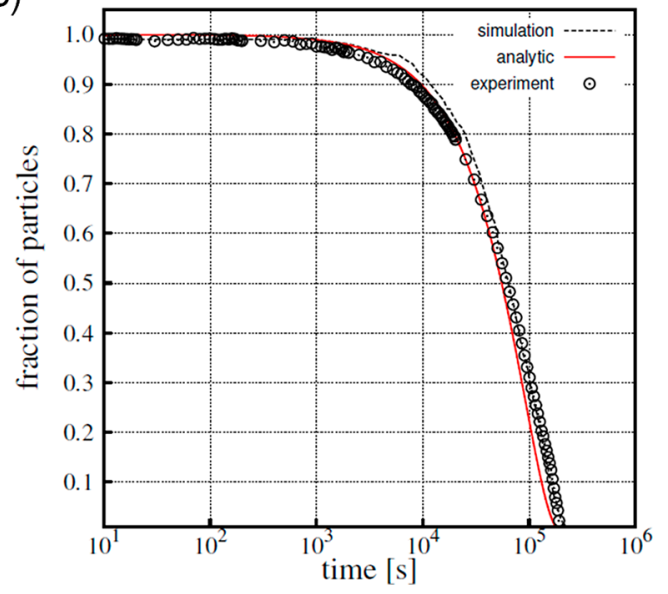

Figure 3. (a) Scheme of a cylindrical magnetic separator for LGMS (top view). See also Figure $1 \mathrm{~b}$ for a picture of an actual separator. (b) Twodimensional plot of the modulus of the magnetic field gradient (in T/m) calculated for a magnetic separator with the geometry indicated in (a) (with $R$ $=1.5 \mathrm{~cm}$ ) employed in the experiments of refs 40 and 71 . The location of the cylindrical vessel of radius $1.5 \mathrm{~cm}$ is indicated by a black line. (c) Comparison of experimental and simulation results and the predictions derived from analytical eq 2 obtained in refs 40 and 71 for an LGMS experiment performed with $\gamma-\mathrm{Fe}_{2} \mathrm{O}_{3}$ nanocrystals of $12 \mathrm{~nm}$ size in the magnetophoretic setup described in (a) and (b).

ization of the particles, with its size and density $\rho_{\mathrm{m}}$ as shown in eq 1. Here, eq 1 implies that the force experienced by a magnetic particle is not in the direction of the magnetic field but in the direction of the magnetic field gradient. The magnetic particles are attracted from the regions with lower magnetic field intensities to the regions with higher magnetic field intensities, with a force proportional to that gradient (in a uniform field $\vec{\nabla} B$ $=0$, so there is no force). Using eq 1 , we can qualitatively analyze the different magnetophoresis examples shown in the Introduction (Figure 1). In Figure 1a, a cylindrical magnet is placed in contact with a vial containing a colloidal suspension of superparamagnetic particles. The field is higher near the wall in physical contact with the cylindrical magnet and decays rapidly with the distance, so such a field gradient causes the particles to move toward the wall close to the magnet. In this case, ${ }^{52,53}$ a typical magnetic field gradient on the order of $\sim 90 \mathrm{~T} / \mathrm{m}$ can be found adjacent to the magnet but drops rapidly below $1 \mathrm{~T} / \mathrm{m}$ over a distance of a few centimeters. In this case, eq 1 predicts a highly inhomogeneous magnetophoretic force, which has important consequences, as we will discuss in detail later. A setup with a uniform magnetic field gradient (and therefore with more homogeneous magnetophoretic conditions) can be obtained with the geometry shown in Figure $1 \mathrm{~b}$ with typical gradients of around $30 \mathrm{~T} / \mathrm{m}$. This setup corresponds to an example of an LGMS magnetic separator. In this design, several magnets are placed in contact with the walls of the vial or bottle containing the suspension, and the particles are attracted to the walls since the magnetic field is zero in the middle and maximum at the walls. As discussed in the Introduction, the setup in Figure 1c shows a completely different strategy based on an HGMS separator column. In this case, an external magnetic field (generated by an electromagnetic coil) was used to magnetize the steel wool, ${ }^{17,27,30}$ and high gradients appear in the vicinity of wool fibers $^{27}$ (typically $10^{2}-10^{4} \mathrm{~T} / \mathrm{m}$ ). These magnetic gradients are so high that magnetic particles passing near the fibers are captured by the magnetophoretic force described by eq 1. It has to be noted that eq 1 has been employed in several modeling works to design and understand HGMS separators. $^{29,32,74-76}$ In the calculations, the trajectories of magnetic particles were obtained by taking into account the magnetic force given by eq 1 in appropriate models of the magnetic fields and the viscous drag. Such calculations gave good agreement with the experimental findings. ${ }^{17,31}$

Equation 1 can also be used as a starting point for the theoretical analysis of the particle motion in LGMS. In particular, the velocity of the particles during magnetophoresis (which we denote as the magnetophoretic velocity) can be obtained by balancing the magnetic force acting on the particle with the opposing viscous drag given by the Stokes law, $\overrightarrow{\mathbf{F}}=$ $6 \pi \eta R_{\mathrm{H}} \overrightarrow{\mathbf{v}}$ (where $\eta$ is the viscosity of the fluid and $R_{\mathrm{H}}$ is the hydrodynamic radius of the particle, assumed to be spherical). Thus, the magnetophoretic velocity can be estimated by

$$
\overrightarrow{\mathbf{v}}=\frac{V_{\mathrm{m}} \rho_{\mathrm{m}}}{6 \pi \eta R_{\mathrm{H}}}|\overrightarrow{\mathbf{M}}(\overrightarrow{\mathbf{B}})| \vec{\nabla} B
$$

Equations 1 and 2 are the bases of the classical model of magnetophoresis. The basic assumption involved in the model is that the interactions between particles (of either magnetic or hydrodynamic origin) are negligible. We will discuss this point in the subsequent subsections.

Equation 2 can be used for a quick estimate of plausible magnetophoretic velocities (for particles of known size using reasonable values of magnetic gradients and magnetizations) and also for modeling the trajectories of particles in a magnetophoretic setup by using particle-level computer simulations. $^{38,71}$ In these simulations, one considers the trajectory of a large number of magnetic particles, distributed in solution inside a magnetic separator by evaluating the velocity of the particles at each point using the detailed profile of the magnetic field distribution (how $\overrightarrow{\mathbf{B}}$ and $\vec{\nabla} B$ depend on position inside the magnetic separator) and the magnetic response $\overrightarrow{\mathbf{M}}=$ $\overrightarrow{\mathbf{M}}(\overrightarrow{\mathbf{B}})$ of the particles.

As a specific example, we consider (Figure 3) the magnetophoretic separation of a dispersion composed of small superparamagnetic nanoparticles $\left(12 \mathrm{~nm} \gamma-\mathrm{Fe}_{2} \mathrm{O}_{3}\right)$ inside a cylindrical magnetic separator with a nearly uniform magnetic field gradient $(|\vec{\nabla} \mathbf{B}| \approx 25-30 \mathrm{~T} / \mathrm{m})$, as reported in ref 40 . The order of magnitude of the magnetophoretic velocity of the particles can be estimated by considering that they have their maximum (saturation) magnetization $\left(M_{s}=68 \mathrm{~A} \mathrm{~m}^{2} / \mathrm{kg}\right)$. Using 
an average density of $\rho_{\mathrm{m}}=4.86 \mathrm{~g} / \mathrm{cm}^{3}$ (see ref 40) and a magnetic field gradient of $\sim 30 \mathrm{~T} / \mathrm{m}$, eq 2 gives $v \approx 0.16 \mu \mathrm{m} / \mathrm{s}$. Therefore, we can estimate that full separation in the cylindrical separator with a radius of $1.5 \mathrm{~cm}$ will require about a day. This is indeed the case, as shown by the experimental results for the fraction of remaining particles as a function of time reported in Figure 3c.

The time evolution of magnetophoresis obtained in experiments is also in full agreement with the predictions obtained from particle-level computer simulations based on eq 2 (dotted line in Figure 3c). These simulations employ as input the calculated magnetic field and magnetic field gradient profiles (Figure 3b), the measured magnetic response of the particles (which depends on the local field), and the measured particle size. The forces acting over the particles in the simulations were the magnetic force given by eq 2 and the viscous drag. It is worth emphasizing that the influence of diffusion is negligible in this case, as shown by the agreement between the simulations (which neglect diffusion) and experiments, as seen in Figure 3c (for a more detailed discussion, see ref 71). It is also possible to calculate the fraction of remaining particles as a function of time using an analytical formula derived in ref 40 (eq 8 in that reference). This expression was derived from eq 2 with the additional assumptions that the magnetic field gradient is uniform and that the magnetic response $\overrightarrow{\mathbf{M}}=\overrightarrow{\mathbf{M}}(\overrightarrow{\mathbf{B}})$ of the particles is given by a classical Langevin function. (See ref 40 for details.) The results are also shown in Figure $3 \mathrm{c}$ and are close to both simulations and experiments.

Finally, to conclude our discussion of the validity of eq 2 , it is interesting that this equation has been generalized to dispersions containing mixtures of magnetic nanoparticles and nonmagnetic colloids, showing excellent agreement with the experimental results. ${ }^{71,77}$ This case is not going to be discussed here in detail, but we emphasize that inside such a mixture the nonmagnetic particles exhibit nontrivial behavior and are moving as magnetic holes (in a direction opposite to that of the magnetic particles). This peculiar phenomenon is also known as negative magnetophoresis. $^{71,77,78}$

The magnetophoretic velocity and the magnetophoretic separation times discussed in the previous example (Figure 3) are obviously too slow to be of practical use in most applications. In view of eq 2, the classical strategy was to resort to the use of high magnetic field gradients as in HGMS such as the setup shown in Figure 1c. However, in the last 12 years, many experimental works ${ }^{10,34,52,53,79,80}$ have systematically reported fast magnetophoretic kinetics under low magnetic gradients, faster than those expected from eq 2 . In fact, the deviation from eq 2 can be several orders of magnitude in many cases, ${ }^{34,52,53}$ indicating the presence of other physical phenomena that contribute to the transport mechanisms of LGMS, which are not being considered by the classical model. The approach followed in the last few years for the research in LGMS has been to investigate these magnetophoretic regimes dominated by effects beyond the classical model, which substantially speed up (by a factor that can be up to $50 \times$ ) the magnetophoretic velocity as compared to the value expected from eq 2 .

Magnetophoresis Beyond the Classical Model. In order to illustrate the characteristics of magnetophoresis beyond the classical model, we show here two prototypical examples (Figure 4). The kinetics observed in both examples clearly departs from the predictions of the classical model (the observed kinetics is much faster than the prediction by eq 2) but also shows very (a)

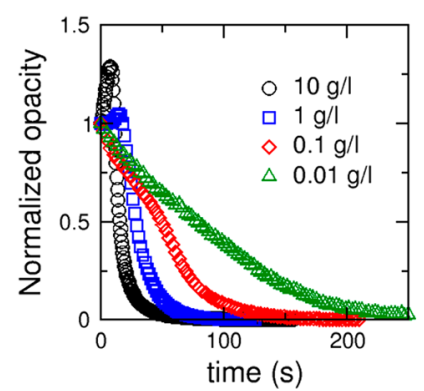

(b)

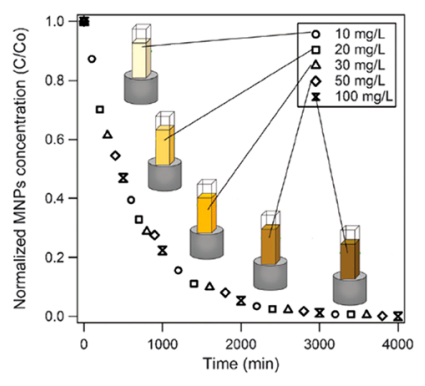

Figure 4. (a) Measured opacity of the dispersion (proportional to the average concentration of particles) as a function of time for water dispersions of Estapor M1-030/40 superparamagnetic beads (diameter of $0.41 \mu \mathrm{m}$ ) during magnetic separation in the same cylindrical uniform magnetic gradient separator employed in the experiments of Figure 3. Reproduced with permission from ref 34. Copyright 2008 American Chemical Society (b). Normalized concentration as a function of time for water solutions of $30 \mathrm{~nm}$ PEG-coated MNP at different concentrations placed on top of a magnet. Reproduced with permission from ref 53. Copyright 2015 The Royal Society of Chemistry.

different characteristic behavior taking place under different conditions originating from very different physical mechanisms.

In Figure $4 \mathrm{a}$, we show results typical for large composite superparamagnetic particles (magnetic "beads") in a uniform magnetic field gradient inside a cylindrical setup such as the separator in Figure $1 \mathrm{~b}$ (in fact, the same as employed in the example of Figure 3). In Figure 4b, we show results typical of functionalized magnetic nanocrystals under the effect of a single magnet, which creates an inhomogeneous magnetic field gradient. In both cases, the kinetics is faster and qualitatively different from that observed in the example of Figure 3. The classical model (eq 2) predicts a separation time ${ }^{34}$ of $\sim 1250 \mathrm{~s}$ for the case in Figure 4a, whereas we observe separations that can be as fast as only $\sim 25 \mathrm{~s}$. (See the curve for the $10 \mathrm{~g} / \mathrm{L}$ concentration in Figure 4a.) Also, the classical model predicts $85000 \mathrm{~min}$ for the case in Figure $4 b,{ }^{53}$ whereas the actual kinetics observed is about 30 times faster. Both experiments also show two striking differences. The first difference is that the magnetophoretic velocity implied by the results in Figure 4a depends on the particle concentration, whereas the results in Figure $4 \mathrm{~b}$ are independent of concentration. The second difference is in the way that the particles disperse within the solution (or concentration profile) during magnetophoresis. In the experiments in Figure $4 a$, the particles and the concentration front advance from the center of the bottle toward the wall, leaving a central region free of particles with a radius that increases as the process advances (for a full video of the process, see ref 14 ). In contrast, for the experiments shown in Figure $4 \mathrm{~b}$, the concentration decreases as a function of time but it is observed to be homogeneous at all times in spite of the fact that the suspension is experiencing a strongly inhomogeneous magnetic field with the strongest magnetic field gradient near to the magnet and almost negligible at the region away from the magnet. This observation is also inconsistent with the classical model.

Cooperative Magnetophoresis. The results in Figure $4 a^{34}$ correspond to the magnetophoretic separation of commercial magnetic beads (Estapor M1-030/40) under the same setup and magnetic field gradient $(\sim 30 \mathrm{~T} / \mathrm{m})$ as employed in the experiments of Figure 3. These particles have a diameter of $\sim 410 \mathrm{~nm}$ and are made by ferrite nanocrystals uniformly 
(a)
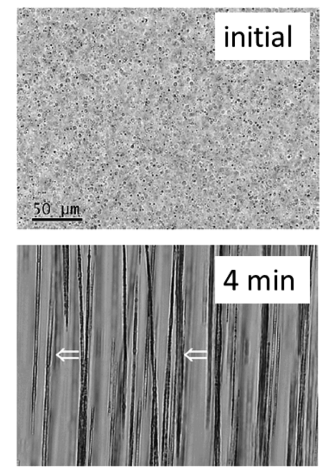

(b)

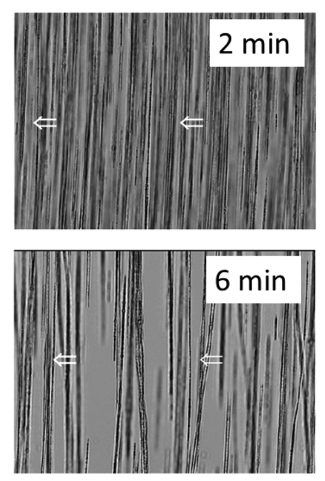

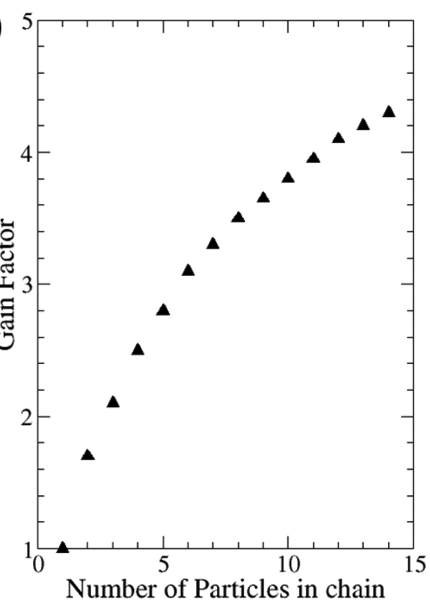

Figure 5. (a) Optical micrograph showing a solution composed of $1 \mathrm{~g} / \mathrm{L}$ Estapor M1-030/40 superparamagnetic beads (diameter of $0.41 \mu \mathrm{m}$ ) under a bar magnet at different times. Linear self-assembled structures form in the direction of the magnetic field and move in the direction of the magnetic field gradient, as indicated by a white arrow. Reproduced with permission from ref 34. Copyright 2008 American Chemical Society. (b) Gain factor for an isolated single chain of $N$ particles (the ratio between the magnetophoretic velocity of a chain of $N$ particles compared with the magnetophoretic velocity of a single particle) as obtained from computer simulations (data adapted from ref 38 ).

distributed inside a polystyrene matrix, and they have a strong superparamagnetic response (saturation magnetization $\sim 45$ $\mathrm{kA} / \mathrm{m}$ ). By using these data in eq 2 , we found that the classical model predicts a magnetophoretic velocity of $v \approx 12 \mu \mathrm{m} / \mathrm{s}$, so the time needed to migrate $1.5 \mathrm{~cm}$ (the radius of the bottle) is predicted to be $\sim 21 \mathrm{~min}^{34}$ The experiments (Figure 4a), however, recorded a separation time of $\sim 25 \mathrm{~s}$ for the highest concentration considered $(10000 \mathrm{mg} / \mathrm{L})$ and a separation time of $\sim 3 \mathrm{~min}$ for the most dilute case $(10 \mathrm{mg} / \mathrm{L})$. Therefore, the actual process as reported in Figure $4 \mathrm{a}$ is much faster than the prediction of eq 2, by a factor of 7 to 50 , depending on the concentration. This effect, mainly originated from the particleparticle interactions, motivated some of us to denote it as cooperative magnetophoresis. ${ }^{34}$ It has been studied experimentally in several subsequent works. ${ }^{19,20,23,34,56,57}$

In order to clarify the origin of this cooperative effect, we have also observed this collective magnetophoretic motion (for the same particles employed in Figure 4a) directly under a microscope. ${ }^{34}$ The movies can also be seen in refs 81 and 82 . We observed the self-assembly of particles into elongated structures with their long axis aligned with the local direction of the magnetic field. These structures are reminiscent of the widely studied case of the formation of chains of particles in the case of homogeneous magnetic fields. ${ }^{35,41,80,83}$ The interesting point here is that these elongated structures move faster than individual particles. They are observed to move in the direction along magnetic field gradient (as dictated by eq 1 ) which has an arbitrary orientation with respect to the direction of the field itself and therefore with respect to the major axis of the structures. This counterintuitive feature is illustrated in the example shown in Figure 5a, which corresponds to the motion of the structures perpendicular to their axis. Of course we have also observed motion in the direction of the major axis, depending on the local orientations of the magnetic field and the magnetic field gradient. ${ }^{34}$ It is also important to recall that this self-assembly process is reversible in the sense that the structures fully dissolve and the dispersion of individual particles is recovered when the magnetic field is removed. ${ }^{34}$

The motion of the self-assembled chain-like structures shown in Figure 5a is faster than the motion of the particles moving as individual (isolated) objects. The structures have a large magnetic dipole moment due to the large number of particles that causes them to experience a large magnetic force (as given by eq 1). In addition, they also experienced a larger friction coefficient than a single particle, but experiments show that the price to pay in friction can be compensated for by the increase in magnetic force. This has also been obtained from simulations by Schaller and co-workers, ${ }^{38}$ as shown in Figure $5 \mathrm{~b}$. In this figure, we show the gain factor (the ratio between the magnetophoretic velocity of a chain of $N$ particles compared with the magnetophoretic velocity of a single particle) which increases as $N$ increases. It should be noted that this gain factor corresponds to a single chain of $N$ particles. This gain is further increased with the lateral aggregation of chains, forming thick bundles of chains as shown in Figure 5a. (The friction of a bundle is similar to that of a chain of the same length, but the magnetic force is increased as more particles are added to the self-assembled structure.) Also, a theoretical analysis based on the balance between magnetic forces and resistance for selfassembled chains made of magnetic particles (ref 50) indicates that the separation time $t$ should decrease as the concentration $c$ increases following a power law:

$$
t \sim c^{-1 / 4}
$$

According to this relationship, speeding up the magnetophoretic separation by a factor of 2 requires an increase in the particle concentration by a factor of $\sim 16$. Equation 3 was found to be in agreement with experimental data for a range of concentrations spanning 3 orders of magnitude (from concentration 0.1 to 10 $\mathrm{g} / \mathrm{L})$. $^{50}$

As a final comment on transport mechanisms for cooperative magnetophoresis, it should be noted that the sedimentation is not usually observed even in the case of long-chain formation. This is due to the low density of the magnetic beads usually employed in these experiments (close to that of water, as we discussed in the previous section) and also to the short times usually involved in cooperative magnetophoretic experiments. Some minor effects of sedimentation were observed during a study of the kinetics of formation of magnetic chains in ref 66 (see also Figure 11 in ref 41 ). In these experiments, composite particles with very high magnetic content and very high density $\left(3.1 \mathrm{~g} / \mathrm{cm}^{3}\right)$ were employed, and some minor effects attributable 
to sedimentation were observed in the absence of magnetophoresis (uniform magnetic field) after long times. Additionally, the trivial effect of gravitational sedimentation on magnetophoresis has also been demonstrated in our experiments using a TODA magnetic nanorod (with a diameter of $\sim 20 \mathrm{~nm}$ and a length of $\sim 300 \mathrm{~nm}) .{ }^{56,84}$ In the presence of a low-gradient magnetic field generated by a hand-held cylindrical magnet, the removal of a magnetic nanorod can be accomplished within 3 min. ${ }^{56}$ On the other hand, under the action of only gravitational force (in the absence of a magnetic field), complete settling out of a magnetic nanorod is still not yet achieved after $100 \mathrm{~min}$ of gravitational sedimentation. ${ }^{84}$ Thus, these experiments have shown the negligible role of gravitational sedimentation in the magnetophoresis of MNP, which will be neglected in the subsequent discussion.

Magnetophoresis-Induced Convection. The example shown in Figure $4 \mathrm{~b}$ demonstrates a completely different effect that also enhances magnetophoresis. In this case, the magnetic interactions between the $30 \mathrm{~nm}$ PEG-coated MNP are too weak to induce self-assembly, as demonstrated by calculations. ${ }^{53}$ Also, experimentally there is no dependency of the magnetophoretic kinetics on concentration since all of the kinetic profiles with different particle concentrations collapsed into a single curve, as shown in Figure $4 \mathrm{~b}$. This observation is contrary to what has been observed in the cooperative magnetophoresis case. The fact that the concentration of particles remains uniform across the entire time scale of the experiment (which decreases as a function of time but is observed to be uniform across the sample) has shed new light on the underlying mechanism of magnetophoresis. As indicated in Figure 6a, the magnetic field (a)

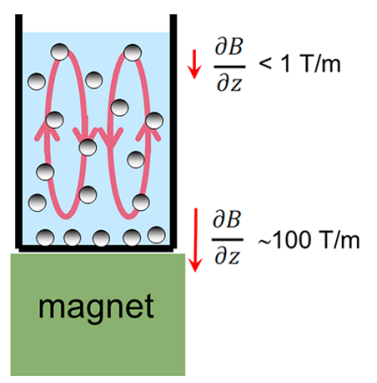

(b)

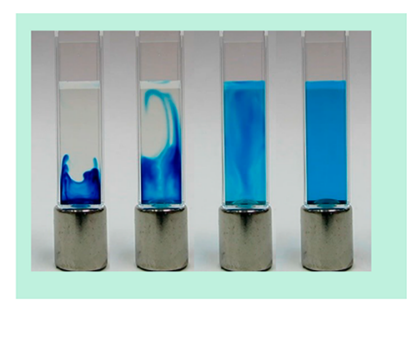

Figure 6. (a) Sketch of a simple setup for observing convection-induced magnetophoresis with a single permanent magnet. Typical values of magnetic field gradients are indicated. The red arrows indicate the spontaneous motion of the fluid. (b) Visualization of convective flow in one of the experiments reported in Figure $4 b$ (a very dilute dispersion of magnetic nanoparticles under the effect of a magnet) using a blue dye. This hydrodynamic instability is induced by a magnet which produces an inhomogeneous magnetic gradient and therefore an inhomogeneous magnetic force, strong in the bottom near the magnet and weak at the top. Reproduced with permission from ref 53. Copyright 2015 Royal Society of Chemistry.

gradient is relatively strong near the magnet (the region where the particles are indeed collected) and quite weak far from it. According to eq 1 , this creates a strongly inhomogeneous force across the fluid, which results in the faster clearance of MNPs in the lower portion of the MNP solution as compared to the upper portion. This would cause the particle concentration in the lower portion to be smaller than that in the upper portion of the solution. When such a solution with inhomogeneous particle concentration (with concentration increasing as one goes from the bottom to the top) is placed within an inhomogeneous magnetic field gradient (with field gradient decreasing with respect to the solution height), a mechanical instability is introduced. In fluids, mechanical instabilities typically relax by inducing convective motions, and this is the case indeed.

As demonstrated by the dye-tracing experiments shown in Figure $6 \mathrm{~b}$, this phenomenon involving the generation of fluid flow due to the magnetophoresis of MNPs can be visually observed quite clearly. ${ }^{53}$ Numerical solutions of the fluid flow equations (Navier-Stokes equations and advection-diffusion equation for the transport of diluted species) including a magnetic term also predict ${ }^{53}$ the generation of a convective motion in the situation of the experiment in Figures $4 \mathrm{~b}$ and 6. This magnetic term takes into account a magnetic body force across the fluid which is proportional to the concentration of magnetic particles and to the force per particle given by eq 1 . The calculations also show that in this case the effect of sedimentation is negligible (see ref 53 for details) so that the magnetic force is the only external field relevant in this convective motion.

In spite of the complexity of the convective problem, the kinetics of magnetophoresis-induced convection as promoted by a single magnet (as in Figures $4 \mathrm{~b}$ and 6 ) can be described by very simple expressions. We have shown both theoretically and experimentally $^{52}$ that the particle concentration (which is uniform across the sample) decreases exponentially with time as $c(t)=c_{0} \exp (-t / \tau)$ with a decay time given by

$$
\tau=\frac{1}{v_{\mathrm{s}}} \frac{V}{A_{\mathrm{s}}}
$$

where $V$ is the volume of the sample, $A_{\mathrm{s}}$ is the area of the vessel in contact with the magnet (the region defined as the collecting surface), and $v_{\mathrm{s}}$ is the magnetophoretic velocity at the collecting surface, evaluated using eq 2 (with the values of magnetization and the magnetic gradient to be employed corresponding to particles at the collecting surface). Here, eq 4 predicts the experimental outcomes with good accuracy (without any fitting parameter $)^{52}$ in many situations, for different particles and different magnetic gradients at the collecting surface.

This convective effect also appears in more complex geometries in which there is also a strong inhomogeneity of the magnetic field gradient. For example, Fratzl and coworkers $^{54}$ have designed a device using a micromagnet array for the fast capture of small nanoparticles which is fully based on this convective effect. They were able to fully capture $12 \mathrm{~nm}$ MNP in only $10 \mathrm{~min}$, a result that until now required the use of HGMS. This demonstrates that particle-fluid interaction is also an integral part of the magnetophoresis.

\section{CHARACTERIZATION OF MAGNETOPHORETIC REGIMES: DIMENSIONLESS NUMBERS}

The results discussed in the previous section demonstrate that both the particle-particle interaction and the particle-fluid interaction are an integral part of magnetophoresis, and they may substantially enhance the kinetics of this process. Our discussion in the previous section has identified two main magnetophoretic regimes of interest: cooperative magnetophoresis $^{34,79,80}$ and magnetophoresis-induced convection. ${ }^{52,53}$ Both scenarios are summarized in Figure 7. Recalling the discussion in the previous section, we can claim that the cooperative magnetophoresis is a collective motion of the particles during their migration process under magnetophoresis 

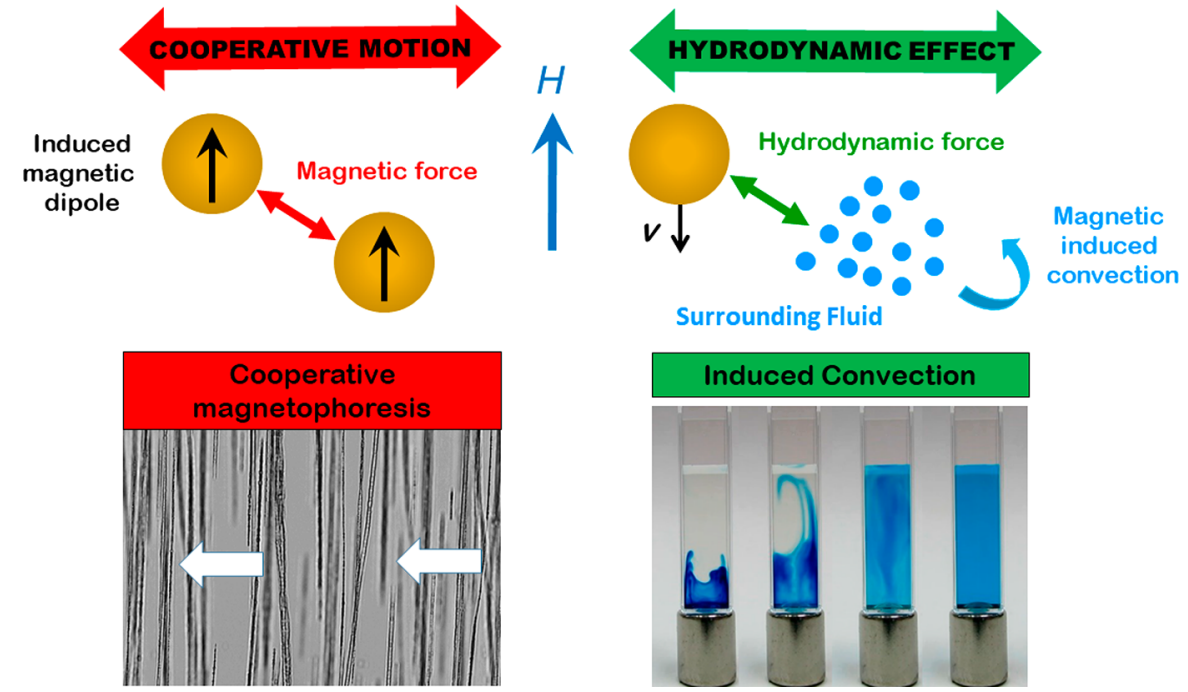

Figure 7. Summary of effects in magnetophoresis due to magnetic and hydrodynamic interactions beyond the classical model. The cartoon indicates the physical interactions behind the cooperative effect and the magnetic-induced convection, illustrated by the images discussed before from the experiments in Figures 5a and 6b.

and is driven by the strong magnetic dipole interaction between the particles. It speeds up the magnetophoresis process in a way that depends crucially on the concentration of magnetic particles. ${ }^{34,35,50}$ Magnetophoresis-induced convection ${ }^{52,53}$ is a hydrodynamic effect that can be observed in the case of highly inhomogeneous magnetic forces over the sample. This occurs when the magnetic field gradient is mostly locally concentrated in a small region (the capture region). In this case, a hydrodynamic instability appears, generating a convective mechanism that substantially speeds up the magnetophoresis process. $^{54}$

In the previous section, we have seen that these effects enhance magnetophoresis in a way that it is described by eqs 3 and 4 for the cases of cooperative and convection effects, respectively. However, a fundamental question here is to determine under which conditions (magnetic field profiles, particle properties, etc.) we will have these mechanisms in operation, which in turn enhances the magnetophoresis. In principle, the problem seems quite complex since in the previous examples there are many factors that are relevant (particle size and magnetization response, concentration, temperature, viscosity, magnetic field gradient magnitude and geometry, etc.). Fortunately, both theoretical and experimental work have demonstrated that all variables relevant to magnetophoresis can be combined into two dimensionless numbers that fully determine the mechanisms at work. The relevant dimensionless numbers are the aggregation parameter, ${ }^{35} N^{*}$, and the magnetic Grashof number, $G r_{\mathrm{m}}$, which correspondingly characterize the emergence of cooperative magnetophoresis and magnetophoresis-induced convection, respectively. ${ }^{53,80}$ They are described in full detail in the remainder of this section.

Aggregation Parameter $N^{*}$. The formation of selfassembled structures such as those shown in Figure 5a depends on the energy minimization involved in the magnetic association but also on the loss of entropy in the solution due to the formation of aggregate structures. The theory behind this process has been reviewed recently. ${ }^{35}$ The main result is that the balance between these thermodynamic factors is given by the dimensionless aggregation parameter $N^{*}$, which involves not only the magnetic properties of the particles but also thermodynamic quantities such as concentration and temperature. $^{35,50,80}$ The critical value of $N^{*}$ for field-induced selfassembly is $N^{*}=1$. In the case of $N^{*}<1$, the formation of structures is not possible (entropy dominates), whereas for $N^{*}>$ 1 , the particles will form elongated structures (simple chains for $N^{*}$ up to 10 and bundles of chains for larger values of $N^{*}$ ). This aggregation parameter can be computed from the parameters of the suspension by ${ }^{35,50,80}$

$$
N^{*}=\sqrt{\phi_{0} \mathrm{e}^{(\Gamma-1)}}
$$

where $\phi_{0}$ is the volume fraction of magnetic particles in the solution (which can be easily computed from its mass concentration) and $\Gamma$ is the magnetic coupling parameter given by ${ }^{85}$

$$
\Gamma=\frac{\mu_{0} m^{2}}{2 \pi d^{3} k_{\mathrm{B}} T}
$$

where $\mu_{0}=4 \pi \times 10^{-7} \mathrm{~N} \mathrm{~A}^{-2}$ is the magnetic permeability of free space, $m$ is the modulus of the magnetic dipole of a particle as induced by the magnetic field (as in eq 1), $d$ is the diameter of the particle, $k_{\mathrm{B}}=1.38 \times 10^{-23} \mathrm{~J} \mathrm{~K}^{-1}$ is Boltzmann's constant, and $T$ is the absolute temperature. Physically, the magnetic coupling parameter $\Gamma$ is the ratio between the magnetic energy of two magnetized particles in contact and the thermal energy. Of course, if thermal energy is stronger than the magnetic interaction $(\Gamma<1)$, then $N^{*}<1$ for any feasible value of the volume fraction and no field induced self-assembly will occur. Values of $\Gamma>1$ imply that the magnetic interaction is stronger than the thermal energy, but self-assembly is not guaranteed since the actual condition for self-assembly $\left(N^{*}>1\right)$ also involves the concentration (eq 5). This is especially relevant in the case of particles with intermediate values of $\Gamma$ (typically below 15) in which self-assembly can be turned on or off with concentration. ${ }^{35,50,80}$ This dependency on concentration becomes irrelevant for the particles that are able to acquire very large values of $\Gamma$, as is often the case for commercial magnetic beads developed for magnetophoresis applications. For an example, in the case of the superparamagnetic beads from 


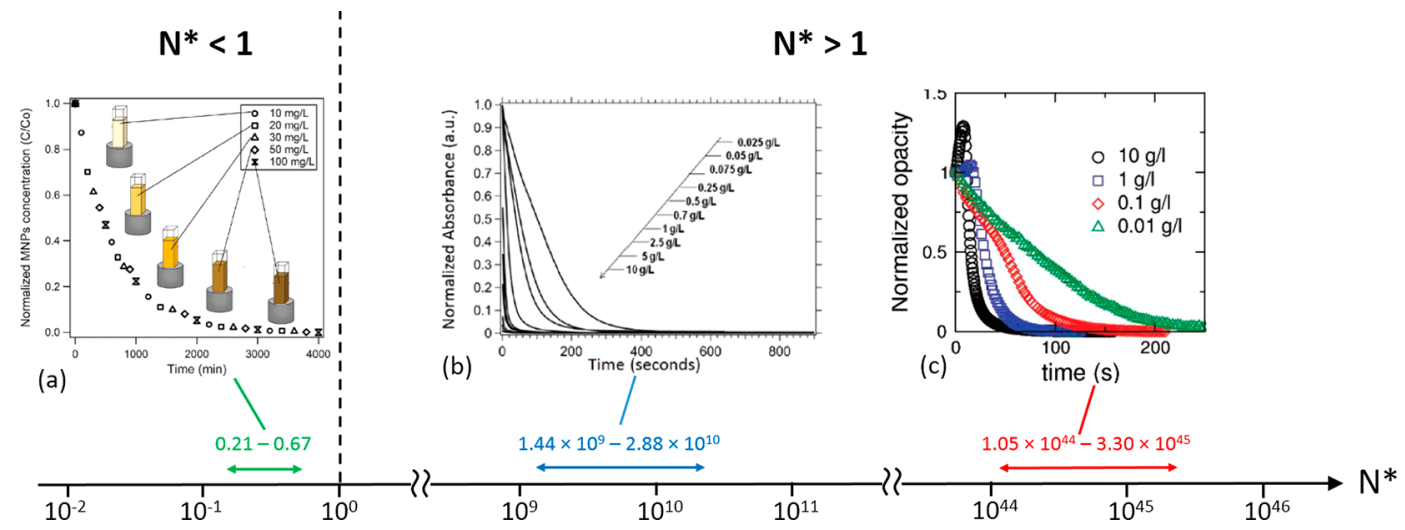

Figure 8. Effect of MNP concentration on the kinetics of magnetophoresis under different values of $N^{*}$ for different particles and experimental setups. (a) When $N^{*}<1$, the magnetophoresis kinetics is independent of concentration, which indicates the absence of particle self-assembly during the magnetophoresis process (noncooperative magnetophoresis). Reproduced with permission from ref 53. Copyright 2015 Royal Society of Chemistry. (b, c) Experimental results from refs 34 and 57 considering different kinds of superparamagnetic particles and setups (small passivated nanoparticles under a simple magnet in (b) and larger composite colloids in a cylindrical setup with a uniform magnetic gradient in (c)). The concentration dependence of these kinetic profiles confirms cooperative magnetophoresis. Reproduced with permission from refs 34 and 57 . Copyright 2014 and 2008 American Chemical Society.

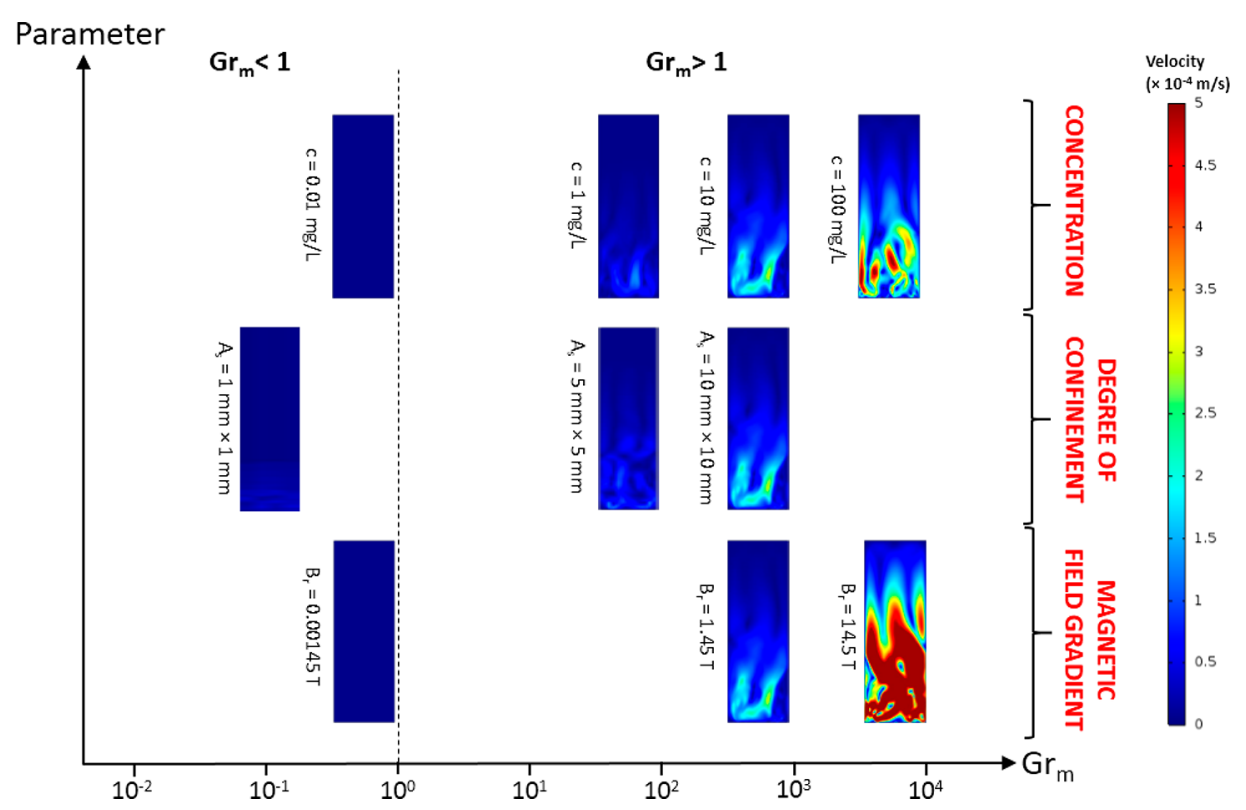

Figure 9. Convective velocity plots in a $2 \mathrm{D} \mathrm{MNP}$ solution (rectangles) subjected to magnetophoresis under different operating conditions (concentration, degree of confinement, and magnetic field gradient). The "magnet" is placed at the bottom of the solutions. The plots are taken $500 \mathrm{~s}$ after the initiation of magnetophoresis. The simulations were carried out using COMSOL Multiphysics. The convection is significant only when the magnetic Grashof number $G r_{\mathrm{m}}$ is greater than unity. The surface plots of $A_{\mathrm{s}}=1 \mathrm{~mm} \times 1 \mathrm{~mm}$ and $A_{\mathrm{s}}=5 \mathrm{~mm} \times 5 \mathrm{~mm}$ (the first two plots in the second row) have been enlarged horizontally such that all surface plots in the figure have the same dimensions for comparison purposes.

the experiment in Figure 4a discussed in the previous section, for $\Gamma \approx 1.8 \times 10^{3}$ we will have $N^{*} \gg 1$ for any feasible concentration. At this point, it should be noted that the concepts leading to eqs 5 and 6 were developed from theory and simulations done in the case of homogeneous magnetic fields. ${ }^{35,41,80}$ In principle, it could be conceivable that in inhomogeneous fields new mechanisms for self-assembly may be possible, which is not considered in the theory of self-assembly under homogeneous fields. This question was addressed in ref 86 , in which selfassembly in homogeneous and inhomogeneous fields (with gradients of about $5 \mathrm{~T} / \mathrm{m}$ ) was compared by detailed nuclear magnetic resonance (NMR) measurements. Interestingly, the results show that the mechanisms for self-assembly are the same in both homogeneous and inhomogeneous fields, with the only difference being the time scales involved in the self-assembly process (which is faster in the presence of a magnetic gradient). Therefore, we conclude that cooperative magnetophoresis will be observed in the presence of a magnetic field gradient when $N^{*}>1$. In agreement with this criterion, reported experimental observations of magnetophoresis (refs 23, 41, 52, 53, 56, 57, 71, 79 , and 80) show cooperative magnetophoresis (with kinetics strongly dependent on concentration) for $N^{*}>1$ and also show noncooperative behavior (kinetics independent of concentration) for $N^{*}<1$. This result is summarized in Figure 8 in which we include results from three key experiments employing different experimental setups and different types of superparamagnetic particles. At this point, the decisive role of particle design in its performance with respect to magnetophoresis is 
worth emphasizing. The design of particles which allow larger values of $N^{*}$, leading to faster magnetophoretic velocities (as seen in Figure 8) even with the modest magnetic field gradients used in simple LGMS, thus making the use of HGMS unnecessary. In fact, this result justifies the tendency of producers of commercial particles to deliver magnetic beads with large sizes and large saturation magnetizations to the market.

Magnetic Grashof Number $\mathrm{Gr}_{\mathrm{m}}$. The second dimensionless number relevant to the behavior of magnetophoresis is the magnetic Grashof number $G r_{\mathrm{m}}$, which is developed to represent the significance of magnetophoresis-induced convection within an MNP solution subjected to an externally applied magnetic field ${ }^{53}$

$$
G r_{\mathrm{m}}=\frac{\rho \nabla B\left(\frac{\partial M}{\partial c}\right)_{H}\left(c_{\mathrm{s}}-c_{\infty}\right) L_{\mathrm{c}}^{3}}{\eta^{2}}
$$

where $\nabla B$ is the magnetic field gradient in the collection surface (the region of the vessel in contact with the permanent magnet), $M$ is the volumetric magnetization of the solution, $c_{\mathrm{s}}$ is the particle concentration at the collection plane, $c_{\infty}$ is the MNP concentration of bulk solution, $L_{\mathrm{c}}$ is the characteristic length of the system subjected to magnetophoresis, and $\rho$ is the mass density of MNP solution. Magnetophoresis-induced convective flow is expected to be relevant if the magnetic Grashof number $G r_{m}$ is greater than unity due to the mechanical instability of the MNP solution. From the microscopic point of view, due to the intensive momentum transfer from moving MNP to the surrounding fluid originating from the viscous effect, the surrounding fluid of the MNP gains momentum and flows convectively, as shown in Figure 7. As a result, in the experiments where MNP solutions are placed on top of a magnet (Figure 8a), this induced flow is found to be more intense for concentrated MNP solutions due to the relatively higher ratio of MNP with respect to the surrounding fluid. ${ }^{53}$ This magnetophoretic-induced convection was also obtained theoretically by numerically solving the fluid flow equations for a $2 \mathrm{D}$ version of the experiment, namely, a solution in a rectangular container under the field created by a magnet. ${ }^{53}$ By extending these calculations, we further report here the results with extensive numerical simulations under different conditions [(i) concentration, (ii) container size (degree of confinement), and (iii) magnetic field gradient] and compare the velocity profiles generated with the corresponding magnetic Grashof numbers. As illustrated in Figure 9, it becomes apparent that the magnetophoresis-induced convection is absent when $G r_{\mathrm{m}}<1$ while the intensity of convection increases with the value of the magnetic Grashof number. The magnitude of the magnetic Grashof number is higher when (i) the MNP solution is more concentrated, (ii) there is less geometrical confinement (commensurate with a larger characteristic length $L_{c}$ ), or (iii) there is a higher magnetic field gradient. (This relationship also can be seen in eq 7.)

It is noteworthy to highlight that the effects of geometrical confinement on the flow behavior of MNP solution were demonstrated indirectly in the simulation results of the magnetophoresis process conducted in continuous flow microfluidic devices (with a length scale on the order of $10^{-4} \mathrm{~m}$ ). The simulation results show only a slight distortion of the parallel layer-by-layer laminar flow of MNP solution in the microfluidic channel, in which vortices are barely observed in the vicinity of the magnets. ${ }^{45,48,49,87}$ On the other hand, the degree of turbulence is more pronounced when magnetophoresis is performed in standard disposable cuvette on a much larger length scale (dimensions $=3 \mathrm{~cm} \times 1 \mathrm{~cm} \times 1 \mathrm{~cm}$ ), in which a chaotic flow pattern is observed, leading to the continuous agitation of the MNP solution, as demonstrated in our experimental and simulation results. ${ }^{52,53}$ Again, this observation is qualitatively consistent with our theoretical prediction based on eq 7, as the magnitude of the magnetic Grashof number (and, hence, intensity of induced convection) increases as the characteristic length increases.

\section{UNIFIED CRITERIA FOR DETERMINING THE ONSET OF COOPERATIVE MAGNETOPHORESIS AND INDUCED CONVECTION IN LGMS}

Magnetophoresis Can Be Classified into Four Different Regimes. In the previous section, we discussed the role of dimensionless numbers $N^{*}$ and $G r_{\mathrm{m}}$ in predicting the presence or absence of magnetically induced particle aggregation and/or magnetophoresis-induced convection. These results as summarized in Figures 8 and 9 can be further condensed into the unified plot shown in Figure 10. In this plot, we can define four

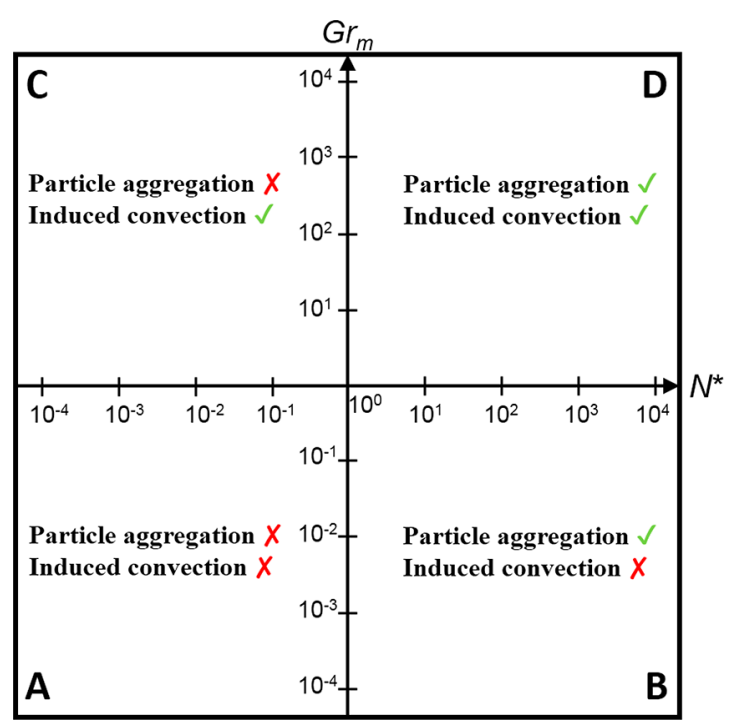

Figure 10. Plot of $G r_{\mathrm{m}}$ against $N^{*}$ for the general magnetophoresis process with four distinct regimes characterized by different flow mechanisms.

different regions according to the values of dimensionless numbers $N^{*}$ and $G r_{\mathrm{m}}$. Depending on the region, the magnetophoresis process occurs via distinctive mechanisms which must be described by different mathematical models.

Region A corresponds to the case with $N^{*}<1$ and $G r_{\mathrm{m}}<1$, so here the classical theory of magnetophoresis can be applied (neither cooperative magnetophoresis nor magnetically induced convection is present). The experiment discussed in Figure 3 corresponds to an example of LGMS performed in this region. Region B $\left(N^{*}>1\right.$ and $\left.G r_{\mathrm{m}}<1\right)$ corresponds to the case of cooperative magnetophoresis (magnetically induced convection is absent here), as in the experiments of Figure 4a. Region C $\left(N^{*}\right.$ $<1$ and $G r_{\mathrm{m}}>1$ ) corresponds to magnetically induced convection, as in the experiments of Figure $4 \mathrm{~b}$. Region D $\left(N^{*}\right.$ $>1$ and $G r_{\mathrm{m}}>1$ ) corresponds to a situation in which both the magnetically induced assembly of particles and magnetically induced convection are present. At the present time, we do not have any reported experimental example of this situation, so it 
remains unexplored. It will be very interesting to explore parameters corresponding to region $\mathrm{D}$ in the future.

In practice, it will be more useful to reconstruct the classification presented in Figure 10 by using accessible parameters such as the axis variables (for example, the particle concentration and magnetic field gradient) instead of working with dimensionless numbers. For the purpose of illustration, in the following subsection we have constructed an explicit example of how to implement the classification shown in Figure 10 in a $2 \mathrm{D}$ plot with the particle concentration and magnetic field gradient as the axis variables. The effect of other design parameters (particle size and magnetization) on the resulting plot is then illustrated in the final subsection. In this regard, it is highly desirable to derive the expressions for threshold values of particle concentration and magnetic field gradient that initiate particle self-assembly and induced convection in terms of the other design parameters.

The condition for magnetically induced self-assembly occurs at $N^{*}>1$, which means that for a given system (i.e., given particle properties) the concentration of particles must exceed the following threshold value (detailed derivation in the Supporting Information)

$$
c>\rho_{\mathrm{p}} \exp \left(1-\frac{\mu_{0} m_{\mathrm{s}}{ }^{2}}{2 \pi d^{3} k_{\mathrm{B}} T}\right)
$$

where $\rho_{\mathrm{p}}$ is the mass density of the particles. The condition for the emergence of magnetophoresis-induced convection $\left(G r_{m}>\right.$ 1) can be transformed into a condition over the magnetic field gradient using eq 7 (the detailed derivation can be found in the Supporting Information)

$$
\nabla B>\frac{\rho_{\mathrm{p}} \eta^{2}}{\rho M_{\mathrm{p}} L_{\mathrm{c}}{ }^{3} \mathrm{c}}
$$

where $M_{\mathrm{p}}$ is the MNP magnetization per unit volume. These results and their physical interpretation are summarized in Table 1.

Representation of the Magnetophoresis Regimes in a Magnetic Field Gradient $\nabla B-$ Concentration c Diagram. The transformation of the classification presented in Figure 10 into a more practical form using the particle concentration and magnetic field gradient as axis variables is illustrated in Figure 11. The tabulations in Figure 11 were evaluated from eqs 8 and 9 by considering a dispersion of $30 \mathrm{~nm}$ magnetite nanoparticles (saturation magnetization of $51.1 \mathrm{~A} \mathrm{~m} \mathrm{~m}^{2} / \mathrm{kg}$ ) coated with poly(ethylene glycol) (PEG). These particles were employed in some of our previous publications ${ }^{52,53}$ and in the experiments shown in Figure 4b.

This $\nabla B-c$ plot provides general guidelines for identifing the physical mechanisms that govern the transport behaviors of magnetophoresis. Furthermore, it allows us to further explore the significance of these control parameters in designing and/or implementing unit operation with the LGMS working principle. For example, using Figure 11 we can see that for these particles the most likely situations are cases $\mathrm{C}$ (magnetically induced convection) and A (classical magnetophoresis). Cases D and B (which involve cooperative magnetophoresis) require concentrations that are too large. For very low concentrations and/or weak magnetic field gradients, we will have case A. For typical magnetic field gradients obtained with a simple hand-held magnet and reasonable concentrations, we will obtain case C. In fact, under the conditions of the magnetic field gradient and

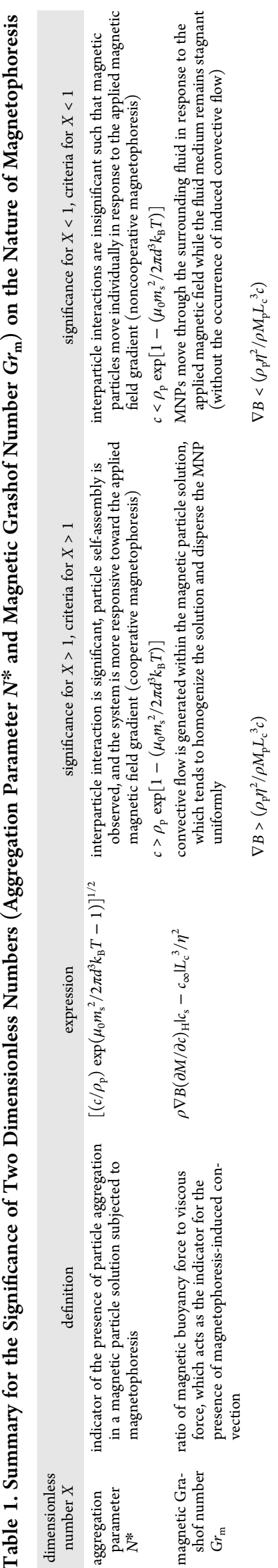




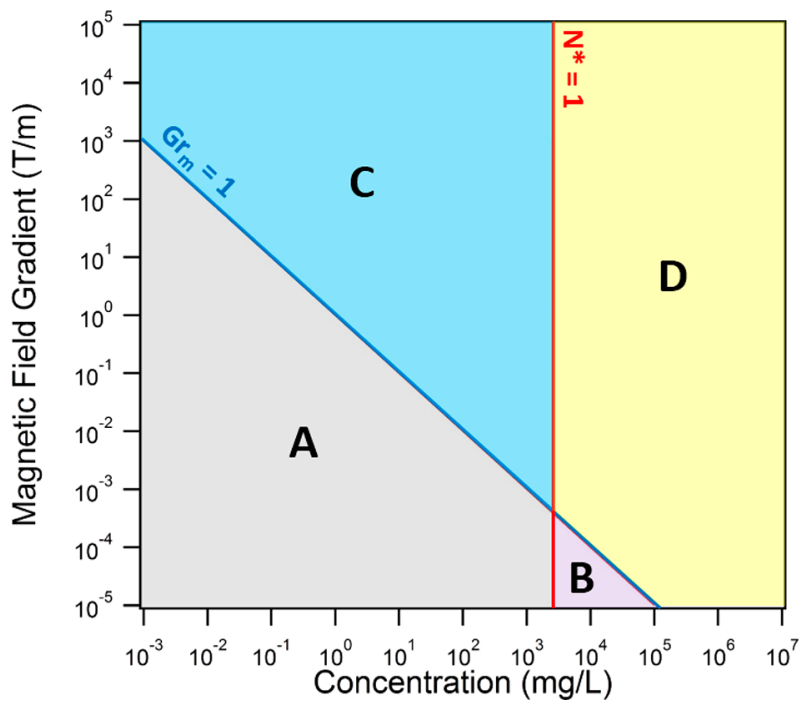

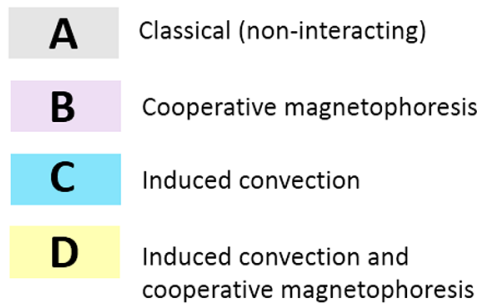

cooperative magnetophoresis

Figure 11. $\nabla B-c$ diagram resulting from the transformation of the classification illustrated in Figure 10 by choosing the particle concentration and magnetic field gradient as axis variables. Labels A-D have the same meaning as in Figure 10. Tabulation of lines $N^{*}=1$ (red) and $G r_{\mathrm{m}}=1$ (blue) in the $\nabla B-c$ plot, which divide the plot into four distinct regions governed by different magnetophoresis models. The reference particle in this calculation is the particle system employed in our previous works, ${ }^{52,53}$ which is a magnetite nanoparticle coated with poly(ethylene glycol) (PEG). The diameter and saturation magnetization of this particle are $30 \mathrm{~nm}$ and $51.1 \mathrm{~A} \mathrm{~m}^{2} / \mathrm{kg}$, respectively.

concentration, the example shown in Figure $4 \mathrm{~b}(\sim 100 \mathrm{~T} / \mathrm{m}$ and concentrations between 10 and $100 \mathrm{mg} / \mathrm{L}$ ) corresponds to case C. Therefore, according to the classification of Figure 11, for such an MNP system, the most convenient design of LGMS should be based on the conditions of magnetically induced convection (case C).

Practical Considerations: Effect of Changing Particle Size and Magnetization. The diagram shown in Figure 11 depends strongly on the size and saturation magnetization of the employed particles. The effect of these parameters is shown in Figure 12 for different particle sizes and magnetizations, typical of MNPs.

The effect of particle size can be clearly seen in Figure 12 . Comparing the plots obtained for different MNPs, we see that as the MNP size increases, line $N^{*}=1$ shifts to the left while line $G r_{\mathrm{m}}=1$ remains unchanged. In other words, the threshold concentration for the onset of particle self-assembly and cooperative magnetophoresis is smaller when larger MNPs are employed. Yellow and purple regions in Figure 12 corresponding to cases $\mathrm{D}$ and $\mathrm{B}$ (cooperative magnetophoresis with and without the convective effect) expand with increasing particle size. The presence or absence of cooperative magnetophoresis is highly sensitive to the particle size. On the contrary, the change in particle size does not have any impact on either the magnetic Grashof number or the location of line $G r_{\mathrm{m}}=1$ in the $\nabla B-c$ plots shown in Figure 12. (Note that the magnetic Grashof number is not dependent on particle size according to eq 7.)

The effect of considering different magnetizations is also seen in Figure 12. As the magnetization of the particle increases, line $N^{*}=1$ shifts to the left while line $G r_{\mathrm{m}}=1$ shifts slightly to the bottom left. The shifting of line $N^{*}=1$ with respect to the change in particle magnetization simply reflects that particles with higher magnetizations have stronger particle-particle interactions (see eq 6 and Table 1) so that the threshold concentration for cooperative magnetophoresis is lower as the particles with higher magnetization are employed. Furthermore, the minor shifting of line $G r_{\mathrm{m}}=1$ indicates that the magnetic field gradient and particle concentration required to generate convective flow within the magnetic particle solution are slightly smaller for a system of particles with greater magnetization. All of these observations suggest strategies for the possible use of MNPs with less magnetocrystallinity (lower magnetization) to achieve the same separation kinetics as obtained for more magnetic particles by taking advantage of the parameters controlling convectively driven magnetic separation. In this scenario, adjusting the magnetic field gradient or using more concentrated dispersions of particles might be a more effective strategy for improving the intensity of convective flow, and it increases the separation kinetics of LGMS.

\section{APPLICATIONS OF MAGNETOPHORESIS UNDER A LOW FIELD GRADIENT}

Applications of LGMS. The ability to remotely manipulate the motion of MNPs by using an externally applied magnetic field is exceptionally appealing in various separation processes in engineering applications, particularly in the environmental treatment and biomedical fields. ${ }^{1,88-90}$ In comparison to conventional separation methods, low-gradient magnetophoresis of MNPs offers various advantages such as simplicity, high adsorption power, high selectivity, high reusability, and material and energy savings. All of these advantages of LGMS are attributed to the recent scientific breakthrough in the synthesis of colloidally stable MNPs (which provide a huge specific surface area for the adsorption process) and various surface functionalization techniques which allow the selective adsorption of targeted entities.

In the field of environmental engineering, MNPs demonstrated excellent performance in the removal of various organic pollutants from an aqueous suspension. ${ }^{91,92}$ Prior to dye removal, MNPs are surface-modified by using specific materials to form nanocomposites which are tailored to the selective adsorption of a targeted dye. ${ }^{93,94}$ For instance, carboxymethyl cellulose-coated $\mathrm{Fe}_{3} \mathrm{O}_{4} @ \mathrm{SiO}_{2}$ core-shell magnetic nanoparticles are synthesized for the efficient removal of a cationic dye (such as methylene blue), ${ }^{95}$ while MNPs copolymerized with catechol and polyethylenimine are found to be effective in the 


\begin{tabular}{|c|c|c|c|}
\hline Magnetization & $20 \mathrm{~nm}$ & $30 \mathrm{~nm}$ & $35 \mathrm{~nm}$ \\
\hline $30 \mathrm{Am}^{2} / \mathrm{kg}$ & 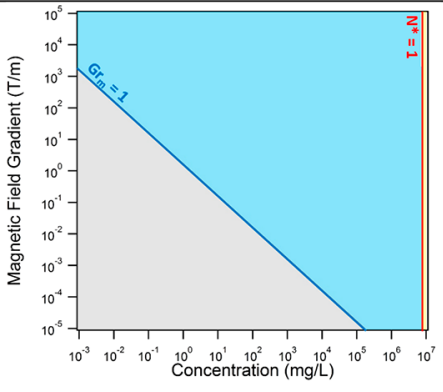 & 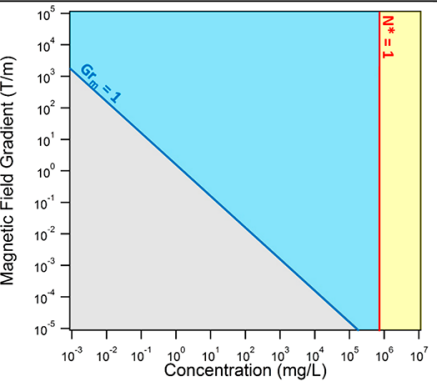 & 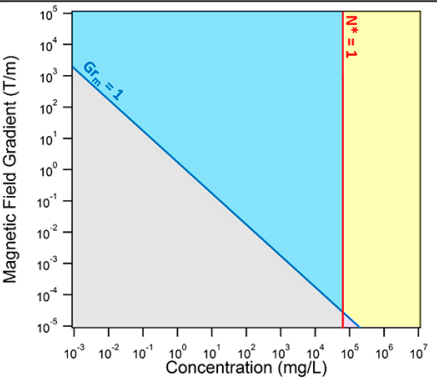 \\
\hline $51.1 \mathrm{Am}^{2} / \mathrm{kg}$ & 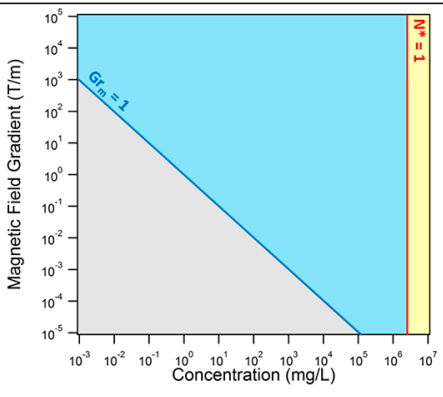 & 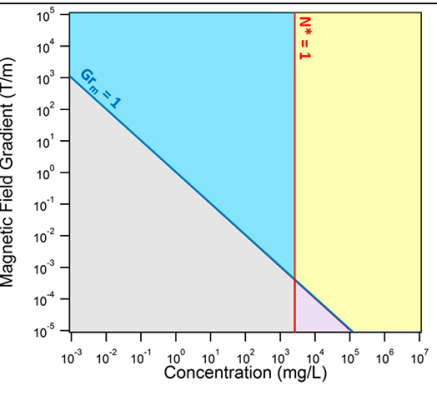 & 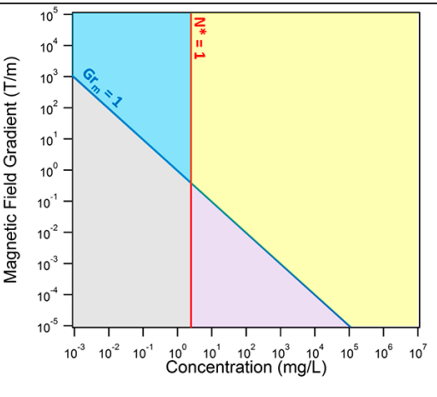 \\
\hline $70 \mathrm{Am}^{2} / \mathrm{kg}$ & 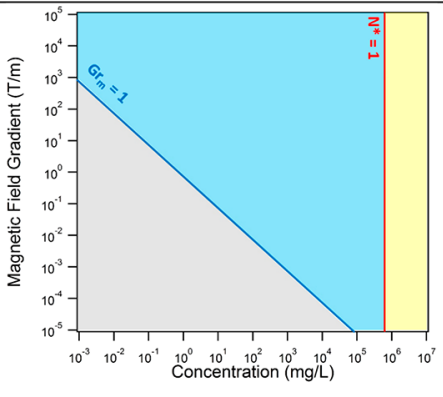 & 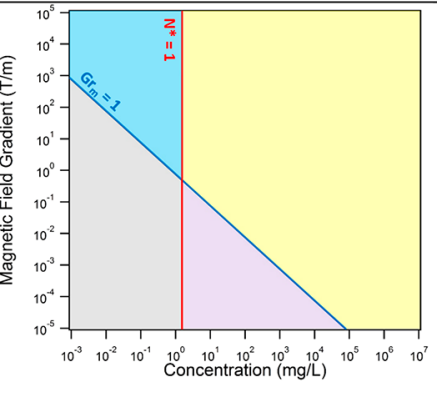 & 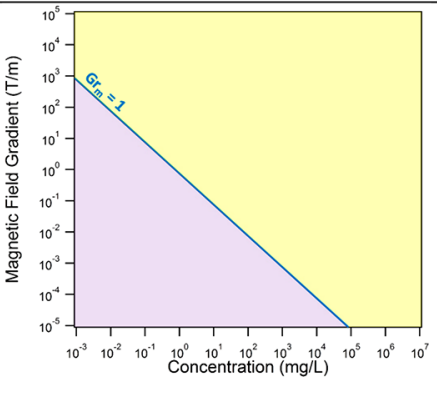 \\
\hline
\end{tabular}

Figure 12. Illustration of the condition changes defined by $N^{*}=1$ and $G r_{\mathrm{m}}=1$ for the $\nabla B-c$ plots with respect to the changes in particle size and magnetization, (See how both lines at $N^{*}=1$ and $G r_{\mathrm{m}}=1$ shifted.) The horizontal and vertical axes of all $\nabla B-c$ plots represent the concentration (in $\mathrm{mg} / \mathrm{L}$ ) and magnetic field gradient (in $\mathrm{T} / \mathrm{m}$ ), respectively. The colors in the diagram represent regions with different separation mechanism, as indicated in Figure 11.

removal of anionic dye from water. ${ }^{96}$ Interestingly, the catalytic degradation and adsorption of dye can also occur simultaneously on the surfaces of MNPs that are functionalized with appropriate materials (Figure 13a,b). ${ }^{97-99}$ It is also noteworthy to highlight that magnetic nanocomposites for dye adsorption also exhibit high reusability, and this feature allows multiple cycle of MNP usage. ${ }^{100,101}$ In addition, surface-modified MNPs are also found to be useful in the removal of various trace heavy metals in aqueous solution, such as selenium, ${ }^{102}$ chromium, ${ }^{103}$ lead, ${ }^{104-107}$ uranium, ${ }^{108}$ mercury, ${ }^{105}$ lanthanide, ${ }^{109}$ copper, $^{101,110}$ iron, ${ }^{111}$ and cadmium. ${ }^{101,106}$ In the petroleum industry, MNPs are also feasible for use in separating oil from water in such a way that MNPs are immobilized onto the oilwater interface to form an $\mathrm{MNP}$-oil emulsion, which further allows the separation of oil from water by using an externally applied magnetic field. ${ }^{12,113}$ Indeed, MNPs appear as a versatile tool in oil adsorption and separation because there are plenty of surface-functioning materials that have been proven to be effective for this purpose, such as amine, ${ }^{114}$ poly(vinylpyrrolidone) (PVP), ${ }^{115}$ (3-aminopropyl)triethoxysilane (APTES), ${ }^{116}$ and chitosan. ${ }^{117}$ Apart from that, MNPs are also effective in harvesting microalgae from water, which can remarkably reduce the biological oxygen demand (BOD) of the treated water. ${ }^{7,118-120}$ Excitingly, Fraga-Garcia and coworkers have demonstrated the successful adsorption of microalgae on a bare iron oxide magnetic nanoparticle without being surface-functionalized. ${ }^{121}$ In contrast to viewing microalgae as pollutants, microalgae are also being treated as green gold for biofuel production (Figure 13c). ${ }^{922,123}$ From this perspective, Liu and co-workers have also developed a rotating drum magnetic separator operated according to the LGMS concept for microalgae separation. ${ }^{124}$

Low-field-gradient magnetophoresis of MNPs also appears to be crucial in the biomedical field. ${ }^{12}$ In particular, the LGMS technique can be employed in diagnosing diseases such as malaria, ${ }^{125,126}$ cancer $^{127}$ and so forth, which is typically accompanied by the detection of the specific biological component in the blood. Since most of the targeted biological components (viruses, bacteria, and pathogens) $)^{8,128,129}$ are eventually nonmagnetic, it is essential to label them with MNPs that have been surface-modified with specific functional groups to allow attachment. ${ }^{12}$ Surface-modified MNPs also acting as the contrast agent in magnetic resonance imaging (MRI) in cancer diagnosis in such a way that the surface- 


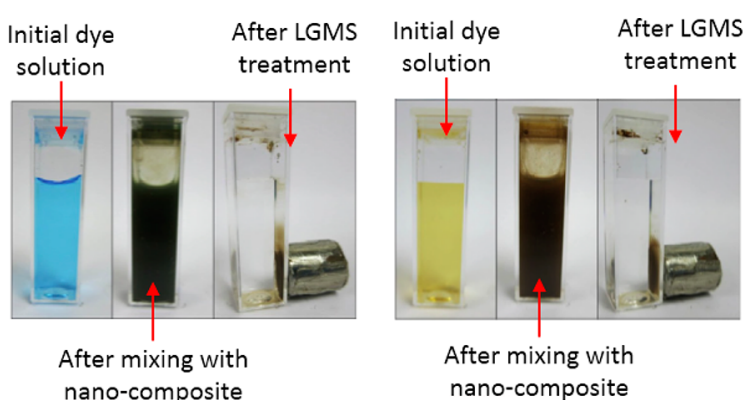

(a) (b)

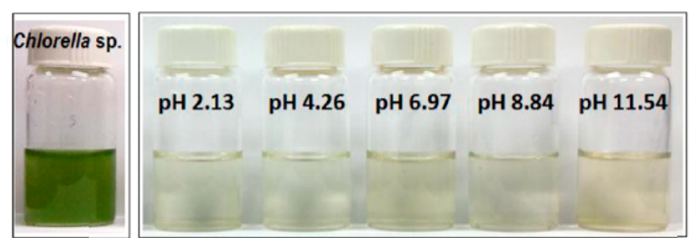

(c)

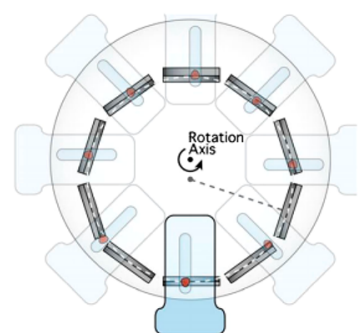

(d)

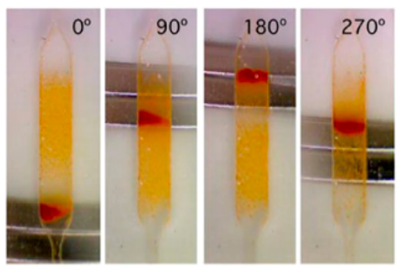

(e)

Figure 13. Successful degradation and removal of dyes (a) methylene blue and (b) methyl orange by using an iron-oxide-MNP/silica composite. Reproduced with permission from ref 99. Copyright 2014 Elsevier. (c) Removal of microalgae (Chlorella sp.) by using PDDA-coated iron oxide MNP. The leftmost photograph shows the initial solution with a high concentration of microalgae. The photographs to the right show the same solution after treatment with the LGMS process at different $\mathrm{pH}$ values. The solutions after LGMS treatment look much clearer (compared with the initial solution), indicating the feasibility of the LGMS technique in removing microalgae over a wide range of $\mathrm{pH}$ values. Reproduced with permission from ref 119 . Copyright 2014 Elsevier. (d) Top view of the magnetic actuator which is designed in such a way that the magnetic beads (MB) within the microfluidic chamber are driven to move back and forth (by a low-gradient magnetic field) when the actuator is rotating. (e) Real-time photographs showing the positions of $\mathrm{MB}$ when the microfluidic chamber is carried to different locations by the magnetic actuator illustrated in (d). Reproduced with permission from ref 137. Copyright 2014 Springer.

\section{Table 2. Properties of Magnetic Particles for Applications as Reported in the Literature}

\begin{tabular}{|c|c|c|c|c|c|}
\hline coating material & particle size $^{a}$ & $\begin{array}{c}\text { particle } \\
\text { concentration }\end{array}$ & $\begin{array}{c}\text { particle } \\
\text { magnetization }^{b}\end{array}$ & application & reference \\
\hline carboxymethyl cellulose (CMC) & $25-40 \mathrm{~nm}$ & $50 \mathrm{mg} / \mathrm{L}$ & $\sim 12 \mathrm{~A} \mathrm{~m}^{2} / \mathrm{kg}$ & methylene blue removal & 95 \\
\hline amine & $10 \mathrm{~nm}\left(d_{\mathrm{H}}=66 \mathrm{~nm}\right)$ & $\begin{array}{l}438-1750 \mathrm{mg} \text { of } \\
\mathrm{Fe} / \mathrm{L}\end{array}$ & $\sim 90 \mathrm{~A} \mathrm{~m}^{2} / \mathrm{kg}$ & oil droplet removal & 114 \\
\hline poly(vinylpyrrolidone) (PVP) & $\begin{array}{l}11.2 \mathrm{~nm} \\
\quad\left(d_{\mathrm{H}}=127.4 \mathrm{~nm}\right)\end{array}$ & $18 \mathrm{mg} / \mathrm{L}$ & & oil removal & 115 \\
\hline $\begin{array}{l}\text { (3-aminopropyl) tiethoxysilane } \\
\text { (APTES) and silica }\end{array}$ & $\begin{array}{l}10 \mathrm{~nm} \\
\left(d_{\mathrm{H}}=105 \mathrm{~nm}\right)\end{array}$ & $100-1300 \mathrm{mg} / \mathrm{L}$ & $40-67 \mathrm{~A} \mathrm{~m}^{2} / \mathrm{kg}$ & emulsified oil removal & 117 \\
\hline hematite & $\begin{array}{l}\sim 10 \mathrm{~nm} \\
\quad\left(d_{\mathrm{H}}=10-30 \mathrm{~nm}\right)\end{array}$ & $100 \mathrm{mg} / \mathrm{L}$ & $27 \mathrm{~A} \mathrm{~m}^{2} / \mathrm{kg}$ & selenium(IV) removal & 102 \\
\hline $\begin{array}{r}\beta \text {-cyclodextrin-ionic liquid } \\
(\beta \text {-CD-IL }) \text { polyurethanes }\end{array}$ & $<50 \mathrm{~nm}$ & $500-5000 \mathrm{mg} / \mathrm{L}$ & $24.6 \mathrm{~A} \mathrm{~m}^{2} / \mathrm{kg}$ & $\begin{array}{l}\text { removal of perfluorooctanesulfonate (PFOS), } \\
\text { perfluorooctanoic acid (PFOA), and } \mathrm{Cr}(\mathrm{VI})\end{array}$ & 103 \\
\hline magnesium oxide $(\mathrm{MgO})$ & $55 \mathrm{~nm}\left(d_{\mathrm{H}}=79 \mathrm{~nm}\right)$ & $100 \mathrm{mg} / \mathrm{L}$ & $20.7 \mathrm{~A} \mathrm{~m}^{2} / \mathrm{kg}$ & lead(II) and methyl orange removal & 104 \\
\hline phosphate-based complex coating & $12 \mathrm{~nm}\left(d_{\mathrm{H}}=32 \mathrm{~nm}\right)$ & & $73.2 \mathrm{~A} \mathrm{~m}^{2} / \mathrm{kg}$ & uranium removal & 108 \\
\hline mercaptoamine and silica & $\begin{array}{l}13.6 \mathrm{~nm} \\
\quad\left(d_{\mathrm{H}}=80 \mathrm{~nm}\right)\end{array}$ & $200-240 \mathrm{mg} / \mathrm{L}$ & $33.4 \mathrm{~A} \mathrm{~m}^{2} / \mathrm{kg}$ & mercury and lead removal & 105 \\
\hline \multirow{2}{*}{$\begin{array}{l}\text { diethylenetriaminepentaacetic } \\
\text { acid }\end{array}$} & $15 \mathrm{~nm}\left(d_{\mathrm{H}}=44 \mathrm{~nm}\right)$ & $8000 \mathrm{mg} / \mathrm{L}$ & & lanthanide ion removal & 109 \\
\hline & $13.1 \mathrm{~nm}$ & & $67 \mathrm{~A} \mathrm{~m}^{2} / \mathrm{kg}$ & microalgae removal & 121 \\
\hline zinc and magnesium & $10-12 \mathrm{~nm}$ & $\sim 200-600 \mathrm{mg} / \mathrm{L}$ & & C. reinharditii and C. vulgaris removal & 122 \\
\hline $\begin{array}{l}\text { poly(diallyldimethylammonium } \\
\text { chloride) (PDDA) }\end{array}$ & $\begin{array}{l}64.4 \mathrm{~nm} \\
\quad\left(d_{\mathrm{H}}=107.4 \mathrm{~nm}\right)\end{array}$ & $20-1000 \mathrm{mg} / \mathrm{L}$ & $71.03 \mathrm{~A} \mathrm{~m}^{2} / \mathrm{kg}$ & removal of microalgae Chlorella sp. & 89 \\
\hline $\begin{array}{l}\text { poly(diallyldimethylammonium } \\
\text { chloride) (PDDA) }\end{array}$ & $20-30 \mathrm{~nm}$ & $300 \mathrm{mg} / \mathrm{L}$ & & $\begin{array}{l}\text { removal of microalgae Chlorella sp. and } \\
\text { Nannochloropsis sp. }\end{array}$ & 120 \\
\hline bovine serum albumin (BSA) & $56 \mathrm{~nm}$ & $\sim 2500 \mathrm{mg} / \mathrm{L}$ & $\sim 20 \mathrm{~A} \mathrm{~m}^{2} / \mathrm{kg}$ & delivery of a curcumin anticancer drug & 65 \\
\hline cyclodextrin & $20 \mathrm{~nm}$ & $\sim 2500 \mathrm{mg} / \mathrm{L}$ & $66 \mathrm{~A} \mathrm{~m}^{2} / \mathrm{kg}$ & delivery of a hydrophobic drug & 133 \\
\hline polyvinyl alcohol (PVA) & $\sim 10 \mathrm{~nm}$ & & $41.78 \mathrm{~A} \mathrm{~m}^{2} / \mathrm{kg}$ & delivery of $5-\mathrm{Fu}$ & 132 \\
\hline anti-E. coli $\mathrm{O} 157: \mathrm{H} 7$ antibody & $2.8 \mu \mathrm{m}$ & $500 \mathrm{mg} / \mathrm{L}$ & $10.8 \mathrm{~A} \mathrm{~m}^{2} / \mathrm{kg}$ & detection of E. coli $\mathrm{O} 157: \mathrm{H7}$ & 137 \\
\hline
\end{tabular}

${ }^{a_{T}}$ The diameters reported here are the core sizes of magnetic nanoparticle. In some cases, the hydrodynamic diameter of the functionalized MNP cluster is shown in parentheses. ${ }^{b}$ The particle magnetization reported here is the magnetization after being functionalized.

modified MNPs are specially designed to concentrate around the region with tumor cells. ${ }^{130,131}$ Additionally, MNPs can also act as drug carriers which transport specified drugs to the desired part of the body. Here, the motion of the MNPs (drug carrier) is manipulated remotely by a magnetic field in a noninvasive manner. For instance, Norasti and co-workers have demon- 
strated that the biocompatible bovine serum albumin (BSA)coated MNPs were effective at delivering a curcumin anticancer drug for chemotherapy. ${ }^{65}$ Owing to all of these features of MNPs in drug delivery, numerous efforts have been dedicated to the synthesis of biocompatible MNPs/magnetic nanocomposites for the delivery of a particular drug to certain targeted parts of the human body for treatment purposes. ${ }^{132-136}$ Another interesting application was the enhancement of detection in immunoassays. ${ }^{137}$ It was found that the use of cooperative lowgradient magnetophoresis enhances the efficiency of an immunoassay designed to detect $E$. coli bacteria with magnetic beads as compared with the efficiency of the same assay performed in the absence of magnetophoresis (Figures 13d,e). This observation was employed to design an improved microfluidic device for immunoassays based on a magnetic actuator and magnetic beads.

In addition, MNPs also play a crucial role in the hyperthermia treatment of cancer in such a way that MNPs are injected into the malignant cells and exposed to an alternating magnetic field which subsequently leads to the release of heat, causing cell death. ${ }^{138,139}$ The essential properties of the MNP system employed in various applications are summarized in Table 2.

Dimensional Analysis of the Separation Mechanism of LGMS in Applications. In all of the applications mentioned in the previous subsection, it is highly desirable to remotely manipulate the motion of MNPs by actuation through an externally applied magnetic field. Typically, for engineering/ biomedical applications that involve the separation of MNPs from the suspension, the separation rate of MNPs is our major concern. On the other hand, for drug delivery or other relevant applications, precise control of the MNP migration pathway and desired destination appears to be the essential factor that determines the practicability of the LGMS technique in these processes. In spite of the different requirements arising from the wide spectrum of applications, understanding the flow behavior and separation mechanism of MNPs under an externally applied magnetic field is equally important for all cases. Thus, in the following paragraphs, we report our findings on performing dimensional analysis on magnetic separation processes operated under engineering relevant conditions, with the focus on the type of application that requires the rapid isolation of MNPs. Such an analysis can provide us a brief overview of the flow mechanism of MNPs in the LGMS system used in engineering processes under the influence of both cooperative and hydrodynamic effects.

In most of the applications involving magnetophoresis, particle concentrations and magnetic field gradients are typically within the ranges of $10-10000 \mathrm{mg} / \mathrm{L}$ and $1-100 \mathrm{~T} / \mathrm{m}, 6,9$ respectively. Such ranges are highlighted within the black-boxed region in the $\nabla B-c$ plots of MNPs at different sizes but with the same magnetization (Figure 14). This boxed region belongs entirely to the area in which the hydrodynamic effect dominates the MNP's flow behavior regardless of the particle size. In other words, the convective flow is always present and almost unavoidable in magnetic separation processes for engineering purposes. Thus, engineers who are working on the design and optimization of magnetic separators for MNP separation should be aware of the presence of magnetically induced convection (unless the magnetic field gradient is perfectly uniform) and should take full advantage of it to accelerate the separation of MNPs from the suspension. Here, the hydrodynamic effect demonstrates a great potential to offset one of the major disadvantages of the magnetic separation technique, namely, the
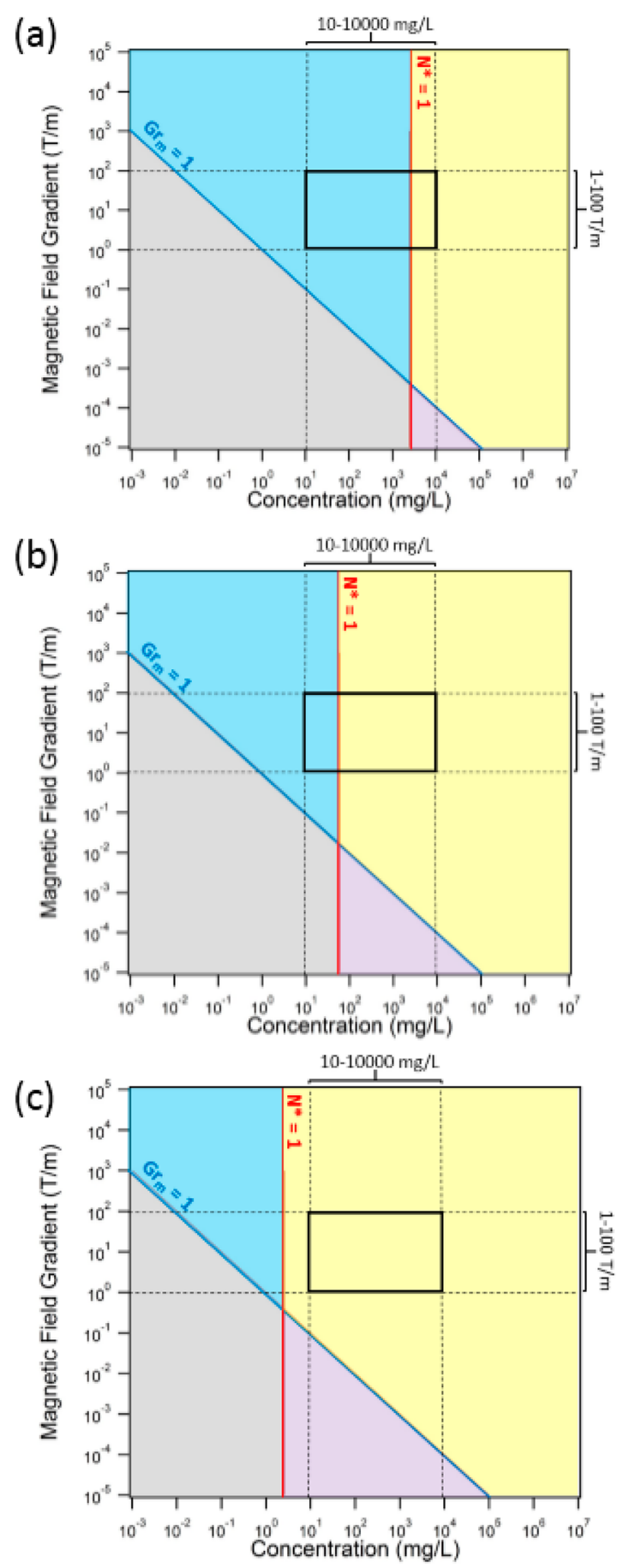

Figure 14. Tabulation of lines $N^{*}=1$ and $G r_{\mathrm{m}}=1$ on the $\nabla B-c$ diagram for particles with the same magnetization $\left(51.1 \mathrm{~A} \mathrm{~m}^{2} / \mathrm{kg}\right)$ but different sizes: (a) 30, (b) 33, and (c) $35 \mathrm{~nm}$. The black-frame square indicates the practical region in which the ranges of the particle concentration and magnetic field gradient are given by $10-10000 \mathrm{mg} /$ $\mathrm{L}$ and $1-100 \mathrm{~T} / \mathrm{m}$, respectively.

low spatial resolution of the magnetic field. ${ }^{13,36,52}$ As the separation distance increases from the magnetic source, the magnetic field is exceptionally weak, which subsequently leads to the inefficient magnetic pulling of MNPs. However, induced convection exhibits a long-range effect, and the convective flow is still sufficiently intense (as compared to individual magnetophoretic velocity) to sweep MNPs in this region toward the magnetic source. As an outcome, the MNPs can be captured on a 
reasonable time scale. ${ }^{53}$ Thus, with the aid of induced convection, a large-scale magnetic separator can be realized in such a way that the separation chamber can be further divided into two distinct zones. The first zone is the region in the vicinity of the magnetic source where the magnetic pulling of MNPs is intensive. The second zone is the region further away from the magnetic source where hydrodynamically induced convection dominates the magnetophoretic force and the motion of MNPs in this zone is mainly driven by the sweeping convective flow. By taking advantage of the hydrodynamic effect, the spatial limitation of the generated magnetic field can be overcome, which subsequently brings a large-scale magnetic separator into the realm of reality.

On the other hand, the presence of the interparticle magnetic dipole-dipole interaction in the black-boxed region varies significantly with respect to the particle size (Figure 14). In fact, the existence of a cooperative effect is extremely sensitive to the particle size, as indicated in Figure 14. It is almost always present for composite magnetic particles (as for commercial magnetic beads), but it is mostly absent for superparamagnetic nanoparticles. For instance, for $30 \mathrm{~nm}$ MNPs, most of the area within this box resides within the noninteracting flow region. (The threshold concentration for the occurrence of interparticle interaction is $\sim 2340 \mathrm{mg} / \mathrm{L}$.) Nonetheless, as the particle size increases, line $N^{*}=1$ is shifted to the left so that the magnetophoretic behavior of the MNP is now dictated by interparticle interaction. More specifically, by increasing the particle diameter from 30 to $33 \mathrm{~nm}$, the threshold concentration for particle interaction is significantly reduced to $\sim 47.8 \mathrm{mg} / \mathrm{L}$, which means that even a very dilute MNP solution is still subjected to the influences of interparticle interaction. As the particle size further increases to $35 \mathrm{~nm}$ (the superparamagnetic limit of the magnetite particle), the entire box is shifted into the yellow region, which is characterized by the presence of intensive interparticle magnetic dipole-dipole interaction. From a practical perspective, the cooperative effect is considered to be beneficial owing to the very rapid magnetophoretic collection of MNPs from the fluid. ${ }^{79}$ However, the aggregation of MNPs will lead to the formation of a large cluster with a reduced surface-to-volume ratio. ${ }^{23}$ Thus, there is a conflicting requirement on the extent of particle aggregation associated with the implementation of the LGMS technique in real time applications. ${ }^{23}$ Particle aggregation is advantageous to inducing the rapid separation of MNPs, but it tends to reduce the surface area for specific functionalities of MNPs. In this regard, we anticipated that the ability to manipulate the aggregation state of the MNP system would be crucial to fully exploiting the advantages of LGMS in real-time applications. In the adsorption/reaction stage, particle aggregation should be avoided to maximize the surface area for specific functionalities. However, in the separation stage, particle aggregation should be encouraged such that MNPs can be collected more rapidly. Interestingly, the manipulation of the particle aggregation state can be realized if a superparamagnetic nanoparticle is employed. As discussed in an earlier section, a superparamagnetic nanoparticle is a special type of paramagnetic material in which the magnetization is strongly dependent on the applied magnetic field but is completely lost upon removal of the external magnetic field. ${ }^{32}$ Owing to this unique property, the aggregation state of any superparamagnetic particle system can be controlled by varying the externally applied magnetic field. Therefore, in real-time applications which involve the separation of MNPs, the adsorption/reaction stage should be used in the absence of an external magnetic field (with particles possessing zero magnetization and a low extent of aggregation), followed by the separation phase under the influence of a magnetic field (particles are magnetized and show higher extents of aggregation). Thus, during the separation phase, technologists should realize that they are working in the area in which cooperative magnetophoresis is occurring such that both the separation rate and efficiency can be enhanced.

\section{CONCLUSIONS}

The use of low magnetic field gradients, as generated by permanent magnets, for the separation and extraction of magnetic particles in solution (also known as low-gradient magnetic separation) is a promising technique with many potential applications. We have revised several of these applications in this feature article. Interestingly, the development of this technique was somewhat unexpected since the magnetic field gradients generated by permanent magnets are too small $(<100 \mathrm{~T} / \mathrm{m})$ to produce useful magnetophoretic motion according to classical theory. However, classical theory fails in predicting the kinetics of the magnetic separation processes under many relevant engineering conditions involving low magnetic field gradients.

Our goal in this feature article was to provide a unified description of this low-gradient magnetophoresis separation process. Our description was elaborated on by way of dimensionless analysis supported by the available theoretical and experimental results.

In the first part of this feature article, we revised and analyzed the scope and limitations of the classical theory of magnetophoresis. We discussed key examples of magnetophoresis that reveal the presence of two key physical effects beyond the classical theory of magnetophoresis. These effects are (i) cooperative magnetophoresis (due to the cooperative motion of strongly interacting particles) and (ii) magnetophoresisinduced convection (a fluid dynamics instability originating from inhomogeneous magnetic gradients). Both mechanisms are responsible for a substantial increment of the separation kinetics of magnetophoresis. In the examples discussed in this feature article, we have estimated an enhancement of the kinetics by a factor of 30 to $50 \times$ depending on the situation. Their action can be identified by the presence of characteristic features. Cooperative magnetophoresis can be identified experimentally by the strong dependence on particle concentration observed in the kinetics of magnetophoresis, which can be described to good approximation by a power law (eq 3 ). The presence of magnetophoresis-induced convection can be confirmed by the presence of macroscopic fluid motion (which can be visualized with the use of dyes as tracers) that maintains a uniform concentration of magnetic particles along the sample. In this case, the kinetics of magnetophoresis is characterized by an exponential decay with a decay time given by eq 4 .

The presence or absence of these transport mechanisms in a specific situation depends on particle properties (such as particle size and magnetization), thermodynamic quantities (concentration, temperature, and solvent viscosity), the profile of the external magnetic field, and the container size and geometry. The dependence on all of these factors can be summarized in two dimensionless parameters-the aggregation parameter $N^{*}$ and the magnetic Grashof number $G r_{\mathrm{m}}$ - which characterize the emergence of cooperative magnetophoresis and magnetophoresis-induced convection, respectively. In the second part of this feature article, we have employed these dimensionless 
quantities to present a classification of magnetophoresis into four distinct regimes depending on the values of these two parameters $\left(N^{*}\right.$ and $\left.G r_{\mathrm{m}}\right):(\mathrm{a})$ a regime of validity of classical magnetophoresis $\left(N^{*}<1, G r_{\mathrm{m}}<1\right)$, (b) a regime dominated by cooperative magnetophoresis $\left(N^{*}>1, G r_{\mathrm{m}}<1\right)$, (c) a regime dominated by magnetically induced convection $\left(N^{*}<1, G r_{\mathrm{m}}>\right.$ 1 ), and (d) a complex regime with both cooperative magnetophoresis and magnetically induced convection $\left(N^{*}>1, G r_{m}>\right.$ 1). This classification can be implemented in convenient diagrams in which control parameters such as the magnetic field gradient and concentration can be used as axis variables so that the regions of validity of each regime can be clearly seen.

The analysis of the diagrams with the different magnetophoretic regimes for particles with different properties (different sizes or different magnetic responses) reveals interesting trends. For example, cooperative magnetophoresis is usually expected for larger magnetic particles (such as the so-called magnetic beads), but it is not expected for smaller magnetic nanoparticles. In the case of magnetic nanoparticles, the situation of magnetically induced convection can be easily obtained with an appropriate choice of control parameters (essentially, the magnetic field gradient). Therefore, it was found that induced convection plays a vital role in governing the behavior of LGMS and pervades engineering applications except on the microscale.

One can take advantage of these effects and the analysis based on dimensionless parameters to modify the operating conditions in order to change the magnetophoretic regime and speed up the separation process, that is, to determine the most effective control parameters that can be manipulated to achieve better separation kinetics. In short, this feature article shows a simple and quick dimensionless analysis for identifying the mechanisms governing magnetophoresis under different conditions. This analysis is particularly crucial in understanding the fundamental behavior of the LGMS process in order to facilitate the design and implementation of the LGMS process in engineering applications. Therefore, the theoretical considerations presented in this feature article may have real-life implications in the optimization and design of the environmental engineering and biomedical applications of MNPs which we have briefly discussed here.

\section{ASSOCIATED CONTENT}

\section{s Supporting Information}

The Supporting Information is available free of charge at https://pubs.acs.org/doi/10.1021/acs.langmuir.0c00839.

Detailed derivation of the criteria for the onset of cooperative magnetophoresis and magnetophoresis-induced convection from the formula of aggregation parameter $N^{*}$ and magnetic Grashof number $G r_{\mathrm{m}}$ (PDF)

\section{AUTHOR INFORMATION}

\section{Corresponding Authors}

Jordi Faraudo - Institut de Ciencia de Materials de Barcelona (ICMAB-CSIC, E-08193 Bellaterra, Spain; 이이이.org/00000002-6315-4993; Email: jfaraudo@icmab.es

JitKang Lim - School of Chemical Engineering, Universiti Sains Malaysia, Nibong Tebal 14300, Penang, Malaysia; Department of Physics, Carnegie Mellon University, Pittsburgh, Pennsylvania 15213, United States; (i) orcid.org/0000-0002-3205-1617; Email: chjitkangl@usm.my

\section{Authors}

Sim Siong Leong - Department of Petrochemical Engineering, Faculty of Engineering and Green Technology, Universiti Tunku Abdul Rahman, Kampar 31900, Perak, Malaysia; School of Chemical Engineering, Universiti Sains Malaysia, Nibong Tebal

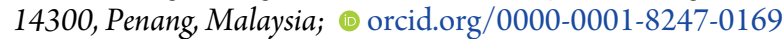

Zainal Ahmad - School of Chemical Engineering, Universiti Sains Malaysia, Nibong Tebal 14300, Penang, Malaysia

Siew Chun Low - School of Chemical Engineering, Universiti Sains Malaysia, Nibong Tebal 14300, Penang, Malaysia; (1) orcid.org/0000-0002-1827-5665

Juan Camacho - Departament de Fisica, Facultat de Ciències, Universitat Autònoma de Barcelona, E-08193 Bellaterra, Spain; (1) orcid.org/0000-0002-8095-4167

Complete contact information is available at:

https://pubs.acs.org/10.1021/acs.langmuir.0c00839

\section{Notes}

The authors declare no competing financial interest.

\section{Biographies}

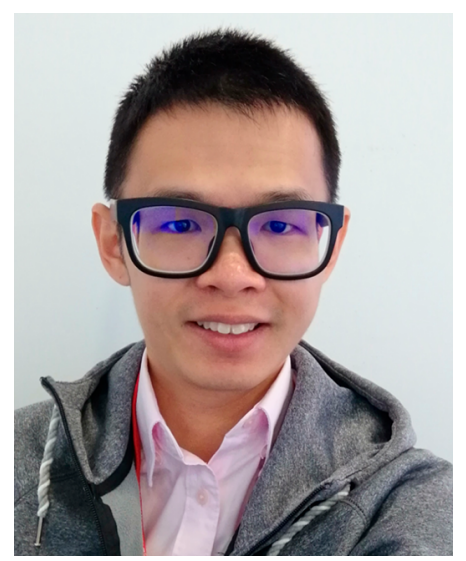

Sim Siong Leong earned his bachelor's degree in chemical engineering at Universiti Sains Malaysia (USM) in 2013. Later, he pursued a Ph.D. degree under the supervision of Prof. JitKang Lim in the School of Chemical Engineering, USM in 2017. Currently, he is holds an assistant professor position in the Petrochemical Engineering Department at Universiti Tunku Adbul Rahman (UTAR). His area of research is nanoscience and nanotechnology, which includes the theoretical modeling of the magnetophoresis of magnetic nanoparticles under low magnetic field gradients and the design of highly efficient magnetic separators to optimize the recollection of magnetic nanoparticles from suspensions.

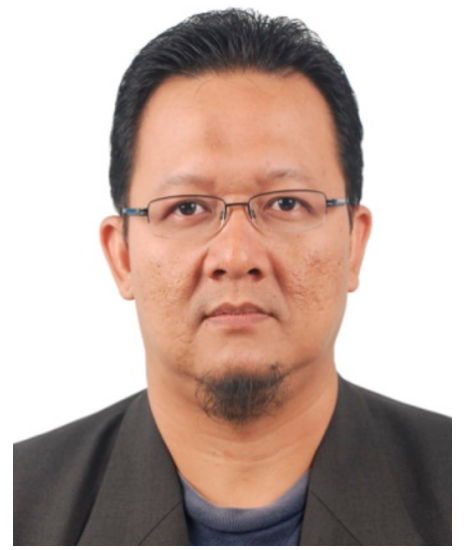

Zainal Ahmad received his diploma in chemical engineering from the University Technology Malaysia in 1996, a BEng (Hons) degree in 
chemical engineering from the University of Surrey, U.K., in 1998, and an MSc (with Distinction) in applied process control in 2001 and a Ph.D. in chemical and process engineering in 2005, both from Newcastle University, U.K.. He has been a professor at the University Sains Malaysia since 2005. His research interests include artificial neural networks, process modeling, model-based process control, and neural network applications in chemical processes. He has guided several master's and Ph.D. students. He has received several awards and has more than 100 international journal/conference publications to his credit.

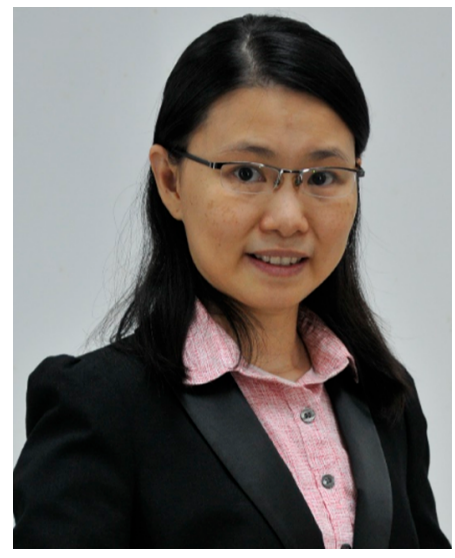

Siew Chun Low received her Ph.D. degree in chemical engineering from Universiti Sains Malaysia (USM) in 2010. Currently, she is an associate professor of chemical engineering at USM, where she is conducting research in the area of wastewater treatment. Siew Chun's research activities are focused on the development of the magnetophoretic-augmented composite membrane in treating polluted river water. She has a particular interest in using magnetic nanoparticles as an antifouling agent which can be incorporated onto the membrane matrix.

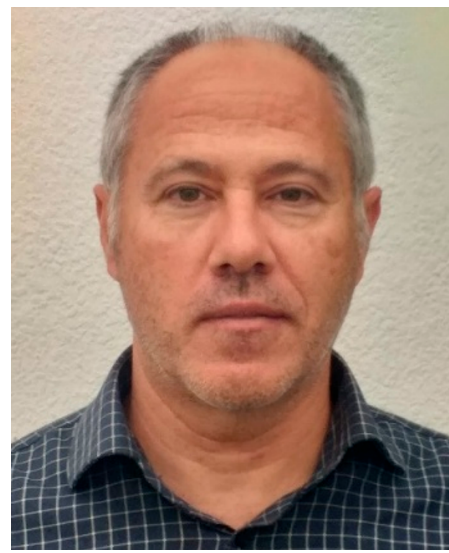

Juan Camacho received his Ph.D. in physics at the Universitat Autònoma de Barcelona in 1992 on the foundations of irreversible thermodynamics. Since 1995, he has been an associate professor of physics at the same university. His research interests include the fields of complexity in biological systems and statistical physics at the nanoscale. Currently, his research is focused on the magnetophoresis and self-aggregation of superparamagnetic suspensions and on thermal transport in nanoscale systems.

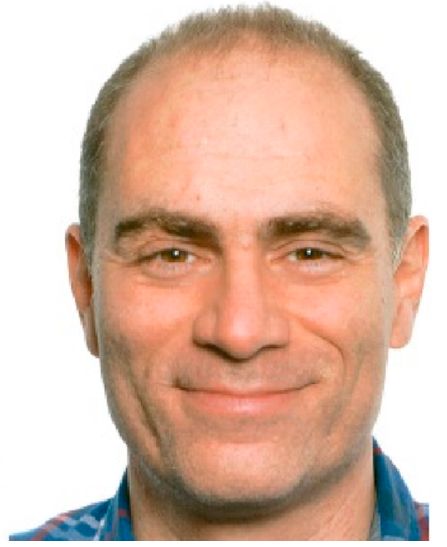

Jordi Faraudo is a tenured scientist at the Theory and Simulation of Materials Department at the Institut de Ciència de Materials de Barcelona (ICMAB-CSIC), being in charge of the soft matter and interfacial science theory research carried out in the department. $\mathrm{He}$ enjoys both developing new simulation tools and applying known simulation tools to new problems motivated by new challenging experiments. His main research interests are self-assembly and selforganization processes arising in nanoparticle dispersions, in supramolecular assemblies (liposomes, vesicles, micelles, etc.), and in molecules at inorganic surfaces. He is also interested in colloidal transport processes such as magnetophoresis and electrophoresis and in fundamental aspects of forces emerging at the nanoscale such as hydration forces, the hydrophobic effect, and colloid forces beyond classical DLVO theory.

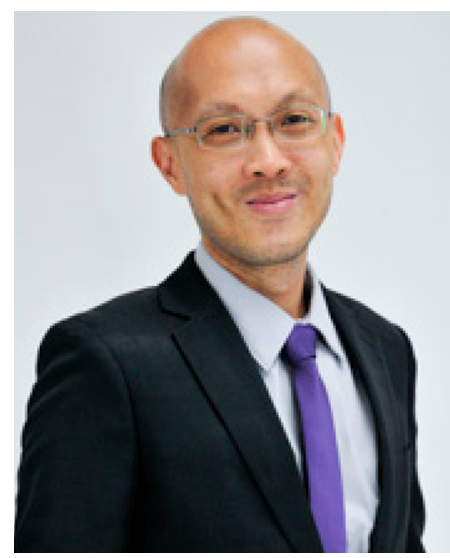

JitKang Lim received his Ph.D. in chemical engineering from Carnegie Mellon University in 2009. Currently, he is a professor of chemical engineering at Universiti Sains Malaysia. His research interest is mainly focused on complex fluids engineering, specifically on the transport and colloidal behavior of magnetic nanomaterials. His most recent research activities include the magnetophoretic control of microrobots, the design of low-gradient magnetic separation systems, and transport phenomena of magnetic nanomaterials under magnetophoresis.

\section{ACKNOWLEDGMENTS}

The authors thank Lluis M. Martinez of Sepmag Systems SL for sharing images of magnetic separators and for invaluable discussions during the last few years about magnetic separation. We also thank Fernando Martinez-Pedrero (UB) for sharing images of his samples. J.L.'s work is financially supported by an LRGS grant from the Ministry of Education Malaysia (project number 67215001). S.S.L. acknowledges the support of UTARRF (project number IPSR/RMC/UTARRF/2019-C2/ L04). J.C. acknowledges financial support by Spain's Ministerio 
de Ciencia, Innovación y Universidades under grant no. RTI2018-097876-B-C22 (MCIU/AEI/FEDER, UE). J.F. is supported by the Spanish Ministry of Science and Innovation through research grant RTI2018-096273-B-I00 and the Severo Ochoa Programme for Centres of Excellence in R\&D (SEV2015-0496) awarded to ICMAB.

\section{REFERENCES}

(1) Zborowski, M.; Sun, L.; Moore, L. R.; Stephen Williams, P.; Chalmers, J. J. Continuous Cell Separation Using Novel Magnetic Quadrupole Flow Sorter. J. Magn. Magn. Mater. 1999, 194 (1), 224230.

(2) Kim, E. J.; Lee, C. S.; Chang, Y. Y.; Chang, Y. S. Hierarchically Structured Manganese Oxide-Coated Magnetic Nanocomposites for the Efficient Removal of Heavy Metal Ions from Aqueous Systems. ACS Appl. Mater. Interfaces 2013, 5 (19), 9628-9634.

(3) Kera, N. H.; Bhaumik, M.; Pillay, K.; Ray, S. S.; Maity, A. Selective Removal of Toxic $\mathrm{Cr}(\mathrm{VI})$ from Aqueous Solution by Adsorption Combined with Reduction at a Magnetic Nanocomposite Surface. J. Colloid Interface Sci. 2017, 503, 214-228.

(4) Pamme, N.; Wilhelm, C. Continuous Sorting of Magnetic Cells via On-Chip Free-Flow Magnetophoresis. Lab Chip 2006, 6 (8), 974-980.

(5) Lakshmanan, R.; Sanchez-Dominguez, M.; Matutes-Aquino, J. A.; Wennmalm, S.; Kuttuva Rajarao, G. Removal of Total Organic Carbon from Sewage Wastewater Using Poly(Ethylenimine)-Functionalized Magnetic Nanoparticles. Langmuir 2014, 30 (4), 1036-1044.

(6) Gómez-Pastora, J.; Bringas, E.; Ortiz, I. Recent Progress and Future Challenges on the Use of High Performance Magnetic NanoAdsorbents in Environmental Applications. Chem. Eng. J. 2014, 256, 187-204.

(7) Toh, P. Y.; Yeap, S. P.; Kong, L. P.; Ng, B. W.; Chan, D. J. C.; Ahmad, A. L.; Lim, J. K. Magnetophoretic Removal of Microalgae from Fishpond Water: Feasibility of High Gradient and Low Gradient Magnetic Separation. Chem. Eng. J. 2012, 211-212, 22-30.

(8) Chen, Y.; Xianyu, Y.; Wang, Y.; Zhang, X.; Cha, R.; Sun, J.; Jiang, $\mathrm{X}$. One-Step Detection of Pathogens and Viruses: Combining Magnetic Relaxation Switching and Magnetic Separation. ACS Nano 2015, 9 (3), 3184-3191.

(9) Song, E. Q.; Hu, J.; Wen, C. Y.; Tian, Z. Q.; Yu, X.; Zhang, Z. L.; Shi, Y. B.; Pang, D. W. Fluorescent-Magnetic-Biotargeting Multifunctional Nanobioprobes for Detecting and Isolating Multiple Types of Tumor Cells. ACS Nano 2011, 5 (2), 761-770.

(10) Yavuz, C. T.; Mayo, J. T.; Yu, W. W.; Prakash, A.; Falkner, J. C.; Yean, S.; Cong, L.; Shipley, H. J.; Kan, A.; Tomson, M.; Natelson, D.; Colvin, V. L. Low-Field Magnetic Separation of Monodisperse Fe3O4 Nanocrystals. Science (Washington, DC, U. S.) 2006, 314 (5801), 964967.

(11) Xu, P.; Zeng, G. M.; Huang, D. L.; Feng, C. L.; Hu, S.; Zhao, M. H.; Lai, C.; Wei, Z.; Huang, C.; Xie, G. X.; Liu, Z. F. Use of Iron Oxide Nanomaterials in Wastewater Treatment: A Review. Sci. Total Environ. 2012, 424, 1-10.

(12) Leong, S. S.; Yeap, S. P.; Lim, J. K. Working Principle and Application of Magnetic Separation for Biomedical Diagnostic at Highand Low-Field Gradients. Interface Focus 2016, 6 (6), 20160048.

(13) Lim, J.; Yeap, S. P.; Low, S. C. Challenges Associated to Magnetic Separation of Nanomaterials at Low Field Gradient. Sep. Purif. Technol. 2014, 123, 171-174.

(14) sepmag. Sepmag: Homogeneous Biomagnetic Separation; https://www.youtube.com/watch?v=BVipEdKoMh8.

(15) López-López, M. T.; Kuzhir, P.; Lacis, S.; Bossis, G.; GonzálezCaballero, F.; Durán, J. D. G. Magnetorheology for Suspensions of Solid Particles Dispersed in Ferrofluids. J. Phys.: Condens. Matter 2006, 18 (38), S2803-S2813.

(16) Rosensweig, R. E. Ferrohydrodynamics; Dover Books on Physics; Dover Publications, 2013.

(17) Moeser, G. D.; Roach, K. A.; Green, W. H.; Hatton, T. A.; Laibinis, P. E. High-Gradient Magnetic Separation of Coated Magnetic Nanoparticles. AIChE J. 2004, 50 (11), 2835-2848.
(18) Wu, W.; He, Q.; Jiang, C. Magnetic Iron Oxide Nanoparticles: Synthesis and Surface Functionalization Strategies. Nanoscale Res. Lett. 2008, 3 (11), 397-415.

(19) Benelmekki, M.; Montras, A.; Martins, A. J.; Coutinho, P. J. G.; Martinez, L. M. Magnetophoresis Behaviour at Low Gradient Magnetic Field and Size Control of Nickel Single Core Nanobeads. J. Magn. Magn. Mater. 2011, 323 (15), 1945-1949.

(20) Benelmekki, M.; Caparros, C.; Montras, A.; Gonçalves, R.; Lanceros-Mendez, S.; Martinez, L. M. Horizontal Low Gradient Magnetophoresis Behaviour of Iron Oxide Nanoclusters at the Different Steps of the Synthesis Route. J. Nanopart. Res. 2011, 13 (8), 3199-3206.

(21) Laurent, S.; Forge, D.; Port, M.; Roch, A.; Robic, C.; Vander Elst, L.; Muller, R. N. Erratum: Magnetic Iron Oxide Nanoparticles: Synthesis, Stabilization, Vectorization, Physicochemical Characterizations, and Biological Applications (Chemical Reviews (2008) 108 (2064)). Chem. Rev. 2010, 110 (4), 2574.

(22) Shen, L.; Laibinis, P. E.; Alan Hatton, T. Bilayer Surfactant Stabilized Magnetic Fluids: Synthesis and Interactions at Interfaces. Langmuir 1999, 15 (2), 447-453.

(23) Yeap, S. P.; Ahmad, A. L.; Ooi, B. S.; Lim, J. Electrosteric Stabilization and Its Role in Cooperative Magnetophoresis of Colloidal Magnetic Nanoparticles. Langmuir 2012, 28 (42), 14878-14891.

(24) Yeap, S. P.; Toh, P. Y.; Ahmad, A. L.; Low, S. C.; Majetich, S. A.; Lim, J. Colloidal Stability and Magnetophoresis of Gold-Coated Iron Oxide Nanorods in Biological Media. J. Phys. Chem. C 2012, 116 (42), 22561-22569.

(25) Wiogo, H. T. R.; Lim, M.; Bulmus, V.; Yun, J.; Amal, R. Stabilization of Magnetic Iron Oxide Nanoparticles in Biological Media by Fetal Bovine Serum (FBS). Langmuir 2011, 27 (2), 843-850.

(26) Yeap, S. P.; Lim, J. K.; Ooi, B. S.; Ahmad, A. L. Feasibility of Electrostatic-Mediated Post-Functionalization to Induce Long Term Colloidal Stability and Stability After Freeze Drying of Amphoteric Nanoparticles. Colloids Interface Sci. Commun. 2018, 23 (November 2017), 14-20.

(27) Ge, W.; Encinas, A.; Araujo, E.; Song, S. Magnetic Matrices Used in High Gradient Magnetic Separation (HGMS): A Review. Results Phys. 2017, 7, 4278-4286.

(28) Toh, P. Y.; Yeap, S. P.; Kong, L. P.; Ng, B. W.; Chan, D. J. C.; Ahmad, A. L.; Lim, J. K. Magnetophoretic Removal of Microalgae from Fishpond Water: Feasibility of High Gradient and Low Gradient Magnetic Separation. Chem. Eng. J. 2012, 211-212, 22-30.

(29) Chen, H.; Kaminski, M. D.; Rosengart, A. J. 2D Modeling and Preliminary in Vitro Investigation of a Prototype High Gradient Magnetic Separator for Biomedical Applications. Med. Eng. Phys. 2008, 30 (1), $1-8$.

(30) Mariani, G.; Fabbri, M.; Negrini, F.; Ribani, P. L. High-Gradient Magnetic Separation of Pollutant from Wastewaters Using Permanent Magnets. Sep. Purif. Technol. 2010, 72 (2), 147-155.

(31) Ditsch, A.; Lindenmann, S.; Laibinis, P. E.; Wang, D. I. C.; Hatton, T. A. High-Gradient Magnetic Separation of Magnetic Nanoclusters. Ind. Eng. Chem. Res. 2005, 44 (17), 6824-6836.

(32) Hatch, G. P.; Stelter, R. E. Magnetic Design Considerations for Devices and Particles Used for Biological High-Gradient Magnetic Separation (HGMS) Systems. J. Magn. Magn. Mater. 2001, 225 (1-2), $262-276$

(33) Corchero, J. L.; Villaverde, A. Biomedical Applications of Distally Controlled Magnetic Nanoparticles. Trends Biotechnol. 2009, 27 (8), 468-476.

(34) De Las Cuevas, G.; Faraudo, J.; Camacho, J. Low-Gradient Magnetophoresis through Field-Induced Reversible Aggregation. J. Phys. Chem. C 2008, 112 (4), 945-950.

(35) Faraudo, J.; Andreu, J. S.; Calero, C.; Camacho, J. Predicting the Self-Assembly of Superparamagnetic Colloids under Magnetic Fields. Adv. Funct. Mater. 2016, 26 (22), 3837-3858.

(36) Lim, J.; Lanni, C.; Evarts, E. R.; Lanni, F.; Tilton, R. D.; Majetich, S. A. Magnetophoresis of Nanoparticles. ACS Nano 2011, 5 (1), 217226. 
(37) Mandel, K.; Hutter, F. The Magnetic Nanoparticle Separation Problem. Nano Today 2012, 7 (6), 485-487.

(38) Schaller, V.; Kräling, U.; Rusu, C.; Petersson, K.; Wipenmyr, J.; Krozer, A.; Wahnström, G.; Sanz-Velasco, A.; Enoksson, P.; Johansson, C. Motion of Nanometer Sized Magnetic Particles in a Magnetic Field Gradient. J. Appl. Phys. 2008, 104 (9), 093918.

(39) Helseth, L. E.; Skodvin, T. Optical Monitoring of Low-Field Magnetophoretic Separation of Particles. Meas. Sci. Technol. 2009, 20 (9), 095202.

(40) Andreu, J. S.; Camacho, J.; Faraudo, J.; Benelmekki, M.; Rebollo, C.; Martínez, L. M. Simple Analytical Model for the Magnetophoretic Separation of Superparamagnetic Dispersions in a Uniform Magnetic Gradient. Phys. Rev. E-Stat. Nonlinear, Soft Matter Phys. 2011, 84 (2), 021402 .

(41) Andreu, J. S.; Calero, C.; Camacho, J.; Faraudo, J. On-the-Fly Coarse-Graining Methodology for the Simulation of Chain Formation of Superparamagnetic Colloids in Strong Magnetic Fields. Phys. Rev. EStat. Nonlinear, Soft Matter Phys. 2012, 85 (3), 036709.

(42) Furlani, E. P. Magnetophoretic Separation of Blood Cells at the Microscale. J. Phys. D: Appl. Phys. 2007, 40 (5), 1313-1319.

(43) Furlani, E. P. Analysis of Particle Transport in a Magnetophoretic Microsystem. J. Appl. Phys. 2006, 99 (2), 024912.

(44) Furlani, E. P.; Sahoo, Y. Analytical Model for the Magnetic Field and Force in a Magnetophoretic Microsystem. J. Phys. D: Appl. Phys. 2006, 39 (9), 1724-1732.

(45) Khashan, S. A.; Furlani, E. P. Effects of Particle-Fluid Coupling on Particle Transport and Capture in a Magnetophoretic Microsystem. Microfluid. Nanofluid. 2012, 12 (1-4), 565-580.

(46) Chen, H.; Kaminski, M. D.; Ebner, A. D.; Ritter, J. A.; Rosengart, A. J. Theoretical Analysis of a Simple yet Efficient Portable Magnetic Separator Design for Separation of Magnetic Nano/Micro-Carriers from Human Blood Flow. J. Magn. Magn. Mater. 2007, 313, 127-134.

(47) Chen, H.; Bockenfeld, D.; Rempfer, D.; Kaminski, M. D.; Liu, X.; Rosengart, A. J. Preliminary 3-D Analysis of a High Gradient Magnetic Separator for Biomedical Applications. J. Magn. Magn. Mater. 2008, 320, 279-284.

(48) Khashan, S. A.; Furlani, E. P. Scalability Analysis of Magnetic Bead Separation in a Microchannel with an Array of Soft Magnetic Elements in a Uniform Magnetic Field. Sep. Purif. Technol. 2014, 125, 311-318.

(49) Khashan, S. A.; Alazzam, A.; Furlani, E. P. Computational Analysis of Enhanced Magnetic Bioseparation in Microfluidic Systems with Flow-Invasive Magnetic Elements. Sci. Rep. 2015, 4, 5299.

(50) Faraudo, J.; Andreu, J. S.; Camacho, J. Understanding Diluted Dispersions of Superparamagnetic Particles under Strong Magnetic Fields: A Review of Concepts, Theory and Simulations. Soft Matter 2013, 9 (29), 6654-6664.

(51) Darras, A.; Opsomer, E.; Vandewalle, N.; Lumay, G. Effect of Volume Fraction on Chains of Superparamagnetic Colloids at Equilibrium. Eur. Phys. J. E: Soft Matter Biol. Phys. 2019, 42 (9), 123.

(52) Leong, S. S.; Ahmad, Z.; Camacho, J.; Faraudo, J.; Lim, J. K. Kinetics of Low Field Gradient Magnetophoresis in the Presence of Magnetically Induced Convection. J. Phys. Chem. C 2017, 121 (9), 5389-5407.

(53) Leong, S. S.; Ahmad, Z.; Lim, J. K. Magnetophoresis of Superparamagnetic Nanoparticles at Low Field Gradient: Hydrodynamic Effect. Soft Matter 2015, 11 (35), 6968-6980.

(54) Fratzl, M.; Delshadi, S.; Devillers, T.; Bruckert, F.; Cugat, O.; Dempsey, N. M.; Blaire, G. Magnetophoretic Induced Convective Capture of Highly Diffusive Superparamagnetic Nanoparticles. Soft Matter 2018, 14 (14), 2671-2681.

(55) Zhu, G.-P.; Nguyen, N.-T. Rapid Magnetofluidic Mixing in a Uniform Magnetic Field. Lab Chip 2012, 12 (22), 4772-4780.

(56) Lim, J. K.; Yeap, S. P.; Leow, C. H.; Toh, P. Y.; Low, S. C. Magnetophoresis of Iron Oxide Nanoparticles at Low Field Gradient: The Role of Shape Anisotropy. J. Colloid Interface Sci. 2014, 421, 170177.
(57) Yeap, S. P.; Leong, S. S.; Ahmad, A. L.; Ooi, B. S.; Lim, J. On Size Fractionation of Iron Oxide Nanoclusters by Low Magnetic Field Gradient. J. Phys. Chem. C 2014, 118 (41), 24042-24054.

(58) Kittel, C. Theory of the Structure of Ferromagnetic Domains in Films and Small Particles. Phys. Rev. 1946, 70 (11-12), 965-971.

(59) Kittel, C. Physical Theory of Ferromagnetic Domains. Rev. Mod. Phys. 1949, 21 (4), 541-583.

(60) Bean, C. P.; Livingston, J. D. Superparamagnetism. J. Appl. Phys. 1959, 30 (4), S120-S129.

(61) Krishnan, K. M.; Pakhomov, A. B.; Bao, Y.; Blomqvist, P.; Chun, Y.; Gonzales, M.; Griffin, K.; Ji, X.; Roberts, B. K. Nanomagnetism and Spin Electronics: Materials, Microstructure and Novel Properties. J. Mater. Sci. 2006, 41 (3), 793-815.

(62) Kim, D. K.; Zhang, Y.; Voit, W.; Rao, K. V.; Muhammed, M. Synthesis and Characterization of Surfactant-Coated Superparamagnetic Monodispersed Iron Oxide Nanoparticles. J. Magn. Magn. Mater. 2001, 225 (1), 30-36.

(63) Yu, S.; Perálvarez-Marín, A.; Minelli, C.; Faraudo, J.; Roig, A.; Laromaine, A. Albumin-Coated SPIONs: An Experimental and Theoretical Evaluation of Protein Conformation, Binding Affinity and Competition with Serum Proteins. Nanoscale 2016, 8 (30), 14393-14405.

(64) Moya, C.; Escudero, R.; Malaspina, D. C.; de la Mata, M.; Hernández-Saz, J.; Faraudo, J.; Roig, A. Insights into Preformed Human Serum Albumin Corona on Iron Oxide Nanoparticles: Structure, Effect of Particle Size, Impact on MRI Efficiency, and Metabolization. ACS Appl. Bio Mater. 2019, 2 (7), 3084-3094.

(65) Nosrati, H.; Sefidi, N.; Sharafi, A.; Danafar, H.; Kheiri Manjili, H. Bovine Serum Albumin (BSA) Coated Iron Oxide Magnetic Nanoparticles as Biocompatible Carriers for Curcumin-Anticancer Drug. Bioorg. Chem. 2018, 76, 501-509.

(66) Chen, D.-X.; Via, G.; Xu, F.-J.; Navau, C.; Sanchez, A.; Gu, H.-C.; Andreu, J. S.; Calero, C.; Camacho, J.; Faraudo, J. Waiting Time Dependence of T2 of Protons in Water Suspensions of Iron-Oxide Nanoparticles: Measurements and Simulations. J. Appl. Phys. 2011, 110 (7), 073917.

(67) Martínez Pedrero, F. Colloidal Aggregation Induced by an Uniaxial Magnetic Field. Ph.D. Thesis, Universidad de Granada, June 2008.

(68) Taboada, E.; Solanas, R.; Rodríguez, E.; Weissleder, R.; Roig, A. Supercritical-Fluid-Assisted One-Pot Synthesis of Biocompatible Core $(\gamma$-Fe2O3)/Shell(SiO2) Nanoparticles as High Relaxivity T2Contrast Agents for Magnetic Resonance Imaging. Adv. Funct. Mater. 2009, 19 (14), 2319-2324.

(69) Martínez-Pedrero, F.; Tirado-Miranda, M.; Schmitt, A.; CallejasFernández, J. Controlling the Magnetic Filaments Length by Tuning the Particle Interactions. J. Colloid Interface Sci. 2008, 318 (1), 23-28.

(70) Chen, D.-X.; Sanchez, A.; Taboada, E.; Roig, A.; Sun, N.; Gu, H.C. Size Determination of Superparamagnetic Nanoparticles from Magnetization Curve. J. Appl. Phys. 2009, 105 (8), 083924.

(71) Andreu, J. S.; Barbero, P.; Camacho, J.; Faraudo, J. Simulation of Magnetophoretic Separation Processes in Dispersions of Superparamagnetic Nanoparticles in the Noncooperative Regime. J. Nanomater. 2012, 2012, 678581.

(72) Melcher, J. R. Continuum Electromechanics; MIT Press: Cambridge, MA, 1981.

(73) Haus, H. A.; Melcher, J. R. Electromagnetic Fields and Energy; Prentice Hall, 1989.

(74) Akoto, I. Mathematical Modelling of High-Gradient Magnetic Separation Devices. IEEE Trans. Magn. 1977, 13 (5), 1486-1489.

(75) Clarkson, C.; Kelland, D. Theory and Experimental Verification of a Model for High Gradient Magnetic Separation. IEEE Trans. Magn. 1978, 14 (3), 97-103.

(76) Cowen, C.; Friedlaender, F.; Jaluria, R. Single Wire Model of High Gradient Magnetic Separation Processes I. IEEE Trans. Magn. 1976, 12 (5), 466-470.

(77) Benelmekki, M.; Martinez, L. M.; Andreu, J. S.; Camacho, J.; Faraudo, J. Magnetophoresis of Colloidal Particles in a Dispersion of Superparamagnetic Nanoparticles: Theory and Experiments. Soft Matter 2012, 8 (22), 6039-6047. 
(78) Hejazian, M.; Li, W.; Nguyen, N.-T. Lab on a Chip for Continuous-Flow Magnetic Cell Separation. Lab Chip 2015, 15 (4), 959-970.

(79) Faraudo, J.; Camacho, J. Cooperative Magnetophoresis of Superparamagnetic Colloids: Theoretical Aspects. Colloid Polym. Sci. 2010, 288 (2), 207-215.

(80) Andreu, J. S.; Camacho, J.; Faraudo, J. Aggregation of Superparamagnetic Colloids in Magnetic Fields: The Quest for the Equilibrium State. Soft Matter 2011, 7 (6), 2336-2339.

(81) Generation of magnetic beads chain-like structure when a magnetic field is applied; https://www.youtube.com/watch?v= MyTXF0SPWTU.

(82) Dissolution of magnetic beads chain-like structures when magnetic field is removed; https://www.youtube.com/watch?v= QgjGjbESuKY.

(83) Darras, A.; Fiscina, J.; Pakpour, M.; Vandewalle, N.; Lumay, G. Ribbons of Superparamagnetic Colloids in Magnetic Field. Eur. Phys. J. E: Soft Matter Biol. Phys. 2016, 39 (4), 47.

(84) Ng, W. M.; Katiyar, A.; Mathivanan, V.; Teng, X. J.; Leong, S.; Low, S.; Lim, J. Sedimentation Kinetics of Magnetic Nanoparticle Clusters: Iron Oxide Nanospheres vs Nanorods. Langmuir 2020, 36 (19), 5085-5095.

(85) Liu, J.; Lawrence, E. M.; Wu, A.; Ivey, M. L.; Flores, G. A.; Javier, K.; Bibette, J.; Richard, J. Field-Induced Structures in Ferrofluid Emulsions. Phys. Rev. Lett. 1995, 74 (14), 2828-2831.

(86) Heinrich, D.; Goñi, A. R.; Osán, T. M.; Cerioni, L. M. C.; Smessaert, A.; Klapp, S. H. L.; Faraudo, J.; Pusiol, D. J.; Thomsen, C. Effects of Magnetic Field Gradients on the Aggregation Dynamics of Colloidal Magnetic Nanoparticles. Soft Matter 2015, 11 (38), 76067616.

(87) Khashan, S. A.; Furlani, E. P. Coupled Particle-Fluid Transport and Magnetic Separation in Microfluidic Systems with Passive Magnetic Functionality. J. Phys. D: Appl. Phys. 2013, 46 (12), 125002.

(88) Zborowski, M.; Ostera, G. R.; Moore, L. R.; Milliron, S.; Chalmers, J. J.; Schechter, A. N. Red Blood Cell Magnetophoresis. Biophys. J. 2003, 84 (4), 2638-2645.

(89) Lim, J. K.; Chieh, D. C. J.; Jalak, S. A.; Toh, P. Y.; Yasin, N. H. M.; Ng, B. W.; Ahmad, A. L. Rapid Magnetophoretic Separation of Microalgae. Small 2012, 8 (11), 1683-1692.

(90) Lim, J.; Majetich, S. A. Composite Magnetic-Plasmonic Nanoparticles for Biomedicine: Manipulation and Imaging. Nano Today 2013, 8 (1), 98-113.

(91) Che, H. X.; Yeap, S. P.; Osman, M. S.; Ahmad, A. L.; Lim, J. Directed Assembly of Bifunctional Silica-Iron Oxide Nanocomposite with Open Shell Structure. ACS Appl. Mater. Interfaces 2014, 6 (19), 16508-16518.

(92) Che, H. X.; Gwee, S. J.; Ng, W. M.; Ahmad, A. L.; Lim, J. Design of Core-Shell Magnetic Nanocomposite by Using Linear and Branched Polycation as an Ad-Layer: Influences of the Structural and Viscoelastic Properties. Colloids Surf., A 2018, 539, 209-220.

(93) Jin, L.; Zhao, X.; Qian, X.; Dong, M. Nickel Nanoparticles Encapsulated in Porous Carbon and Carbon Nanotube Hybrids from Bimetallic Metal-Organic-Frameworks for Highly Efficient Adsorption of Dyes. J. Colloid Interface Sci. 2018, 509, 245-253.

(94) Babaei, A. A.; Kakavandi, B.; Rafiee, M.; Kalantarhormizi, F.; Purkaram, I.; Ahmadi, E.; Esmaeili, S. Comparative Treatment of Textile Wastewater by Adsorption, Fenton, UV-Fenton and US-Fenton Using Magnetic Nanoparticles-Functionalized Carbon (MNPs@C). J. Ind. Eng. Chem. 2017, 56, 163-174.

(95) Zirak, M.; Abdollahiyan, A.; Eftekhari-Sis, B.; Saraei, M. Carboxymethyl Cellulose Coated Fe 304 @SiO 2 Core-Shell Magnetic Nanoparticles for Methylene Blue Removal: Equilibrium, Kinetic, and Thermodynamic Studies. Cellulose 2018, 25 (1), 503-515.

(96) Long, Y.; Xiao, L.; Cao, Q. Co-Polymerization of Catechol and Polyethylenimine on Magnetic Nanoparticles for Efficient Selective Removal of Anionic Dyes from Water. Powder Technol. 2017, 310, 2434.

(97) Prasad, C.; Yuvaraja, G.; Venkateswarlu, P. Biogenic Synthesis of Fe3O4Magnetic Nanoparticles Using Pisum Sativum Peels Extract and
Its Effect on Magnetic and Methyl Orange Dye Degradation Studies. J. Magn. Magn. Mater. 2017, 424, 376-381.

(98) Kong, L.; Gan, X.; Ahmad bin, A. L.; Hamed, B. H.; Evarts, E. R.; Ooi, B.; Lim, J. Design and Synthesis of Magnetic Nanoparticles Augmented Microcapsule with Catalytic and Magnetic Bifunctionalities for Dye Removal. Chem. Eng. J. 2012, 197, 350-358.

(99) Che, B. H. X.; Yeap, S. P.; Ahmad, A. L.; Lim, J. Layer-by-Layer Assembly of Iron Oxide Magnetic Nanoparticles Decorated Silica Colloid for Water Remediation. Chem. Eng. J. 2014, 243, 68-78.

(100) Guo, R.; Jiao, T.; Li, R.; Chen, Y.; Guo, W.; Zhang, L.; Zhou, J.; Zhang, Q.; Peng, Q. Sandwiched Fe3O4/Carboxylate Graphene Oxide Nanostructures Constructed by Layer-by-Layer Assembly for Highly Efficient and Magnetically Recyclable Dye Removal. ACS Sustainable Chem. Eng. 2018, 6 (1), 1279-1288.

(101) Bharath, G.; Alhseinat, E.; Ponpandian, N.; Khan, M. A.; Siddiqui, M. R.; Ahmed, F.; Alsharaeh, E. H. Development of Adsorption and Electrosorption Techniques for Removal of Organic and Inorganic Pollutants from Wastewater Using Novel Magnetite/ Porous Graphene-Based Nanocomposites. Sep. Purif. Technol. 2017, $188,206-218$.

(102) Ma, Z.; Shan, C.; Liang, J.; Tong, M. Efficient Adsorption of Selenium(IV) from Water by Hematite Modified Magnetic Nanoparticles. Chemosphere 2018, 193, 134-141.

(103) Badruddoza, A. Z. M.; Bhattarai, B.; Suri, R. P. S. Environmentally Friendly $\beta$-Cyclodextrin-Ionic Liquid Polyurethane-Modified Magnetic Sorbent for the Removal of PFOA, PFOS, and Cr(VI) from Water. ACS Sustainable Chem. Eng. 2017, 5 (10), 9223-9232.

(104) Ge, L.; Wang, W.; Peng, Z.; Tan, F.; Wang, X.; Chen, J.; Qiao, X. Facile Fabrication of Fe@MgO Magnetic Nanocomposites for Efficient Removal of Heavy Metal Ion and Dye from Water. Powder Technol. 2018, 326, 393-401.

(105) Bao, S.; Li, K.; Ning, P.; Peng, J.; Jin, X.; Tang, L. Highly Effective Removal of Mercury and Lead Ions from Wastewater by Mercaptoamine-Functionalised Silica-Coated Magnetic Nano-Adsorbents: Behaviours and Mechanisms. Appl. Surf. Sci. 2017, 393, 457-466.

(106) Chen, K.; He, J.; Li, Y.; Cai, X.; Zhang, K.; Liu, T.; Hu, Y.; Lin, D.; Kong, L.; Liu, J. Removal of Cadmium and Lead Ions from Water by Sulfonated Magnetic Nanoparticle Adsorbents. J. Colloid Interface Sci. 2017, 494, 307-316.

(107) Tiwari, S.; Hasan, A.; Pandey, L. M. A Novel Bio-Sorbent Comprising Encapsulated Agrobacterium Fabrum (SLAJ731) and Iron Oxide Nanoparticles for Removal of Crude Oil Co-Contaminant, Lead $\mathrm{Pb}$ (II). J. Environ. Chem. Eng. 2017, 5 (1), 442-452.

(108) Calì, E.; Qi, J.; Preedy, O.; Chen, S.; Boldrin, D.; Branford, W. R.; Vandeperre, L.; Ryan, M. P. Functionalised Magnetic Nanoparticles for Uranium Adsorption with Ultra-High Capacity and Selectivity. J. Mater. Chem. A 2018, 6 (7), 3063-3073.

(109) de Melo, F. M.; Almeida da, S. N.; Santos dos, A. D.; Toma, H. E. Magnetophoresis of Superparamagnetic Nanoparticles Applied to the Extraction of Lanthanide Ions in the Presence of Magnetic Field. NanoWorld J. 2017, 03 (02), 38-43.

(110) Sahraei, R.; Sekhavat Pour, Z.; Ghaemy, M. Novel Magnetic Bio-Sorbent Hydrogel Beads Based on Modified Gum Tragacanth/ Graphene Oxide: Removal of Heavy Metals and Dyes from Water. J. Cleaner Prod. 2017, 142, 2973-2984.

(111) Meng, C.; Zhikun, W.; Qiang, L.; Chunling, L.; Shuangqing, S.; Songqing, H. Preparation of Amino-Functionalized Fe3O4@mSiO2 Core-Shell Magnetic Nanoparticles and Their Application for Aqueous Fe3+ Removal. J. Hazard. Mater. 2018, 341, 198-206.

(112) Simonsen, G.; Strand, M.; Øye, G. Potential Applications of Magnetic Nanoparticles within Separation in the Petroleum Industry. J. Pet. Sci. Eng. 2018, 165, 488-495.

(113) Yang, H.; Zhang, H.; Peng, J.; Zhang, Y.; Du, G.; Fang, Y. Smart Magnetic Ionic Liquid-Based Pickering Emulsions Stabilized by Amphiphilic Fe3O4 Nanoparticles-Highly Efficient Extraction Systems for Water Purification. J. Colloid Interface Sci. 2017, 485, 213-222.

(114) Ko, S.; Kim, E. S.; Park, S.; Daigle, H.; Milner, T. E.; Huh, C.; Bennetzen, M. V.; Geremia, G. A. Amine Functionalized Magnetic 
Nanoparticles for Removal of Oil Droplets from Produced Water and Accelerated Magnetic Separation. J. Nanopart. Res. 2017, 19 (4), 132.

(115) Mirshahghassemi, S.; Ebner, A. D.; Cai, B.; Lead, J. R. Application of High Gradient Magnetic Separation for Oil Remediation Using Polymer-Coated Magnetic Nanoparticles. Sep. Purif. Technol. 2017, 179, 328-334.

(116) Lü, T.; Zhang, S.; Qi, D.; Zhang, D.; Vance, G. F.; Zhao, H. Synthesis of PH-Sensitive and Recyclable Magnetic Nanoparticles for Efficient Separation of Emulsified Oil from Aqueous Environments. Appl. Surf. Sci. 2017, 396, 1604-1612.

(117) Lü, T.; Chen, Y.; Qi, D.; Cao, Z.; Zhang, D.; Zhao, H. Treatment of Emulsified Oil Wastewaters by Using Chitosan Grafted Magnetic Nanoparticles. J. Alloys Compd. 2017, 696, 1205-1212.

(118) Agarwal, P.; Gupta, R.; Agarwal, N. Advances in Synthesis and Applications of Microalgal Nanoparticles for Wastewater Treatment. J. Nanotechnol. 2019, 2019, 7392713.

(119) Toh, P. Y.; Ng, B. W.; Ahmad, A. L.; Chieh, D. C. J.; Lim, J. K. Magnetophoretic Separation of Chlorella Sp.: Role of Cationic Polymer Binder. Process Saf. Environ. Prot. 2014, 92 (6), 515-521.

(120) Toh, P. Y.; Ng, B. W.; Ahmad, A. L.; Chieh, D. C. J.; Lim, J. The Role of Particle-to-Cell Interactions in Dictating Nanoparticle Aided Magnetophoretic Separation of Microalgal Cells. Nanoscale 2014, 6 (21), 12838-12848.

(121) Fraga-García, P.; Kubbutat, P.; Brammen, M.; Schwaminger, S.; Berensmeier, S. Bare Iron Oxide Nanoparticles for Magnetic Harvesting of Microalgae: From Interaction Behavior to Process Realization. Nanomaterials 2018, 8 (5), 292.

(122) Egesa, D.; Chuck, C. J.; Plucinski, P. Multifunctional Role of Magnetic Nanoparticles in Efficient Microalgae Separation and Catalytic Hydrothermal Liquefaction. ACS Sustainable Chem. Eng. 2018, 6 (1), 991-999.

(123) Zhu, L. D.; Hiltunen, E.; Li, Z. Using Magnetic Materials to Harvest Microalgal Biomass: Evaluation of Harvesting and Detachment Efficiency. Environ. Technol. 2019, 40 (8), 1006-1012.

(124) Wang, S.-K.; Stiles, A. R.; Guo, C.; Liu, C.-Z. Harvesting Microalgae by Magnetic Separation: A Review. Algal Res. 2015, 9, 178185.

(125) Ngo, H. T.; Gandra, N.; Fales, A. M.; Taylor, S. M.; Vo-Dinh, T. Sensitive DNA Detection and SNP Discrimination Using Ultrabright SERS Nanorattles and Magnetic Beads for Malaria Diagnostics. Biosens. Bioelectron. 2016, 81, 8-14.

(126) Melville, D.; Paul, F.; Roath, S. Direct Magnetic Separation of Red Cells from Whole Blood. Nature 1975, 255, 706.

(127) Kang, T.; Li, F.; Baik, S.; Shao, W.; Ling, D.; Hyeon, T. Surface Design of Magnetic Nanoparticles for Stimuli-Responsive Cancer Imaging and Therapy. Biomaterials 2017, 136, 98-114.

(128) Chen, G. D.; Alberts, C. J.; Rodriguez, W.; Toner, M. Concentration and Purification of Human Immunodeficiency Virus Type 1 Virions by Microfluidic Separation of Superparamagnetic Nanoparticles. Anal. Chem. 2010, 82 (2), 723-728.

(129) Lien, K.-Y.; Lin, J.-L.; Liu, C.-Y.; Lei, H.-Y.; Lee, G.-B. Purification and Enrichment of Virus Samples Utilizing Magnetic Beads on a Microfluidic System. Lab Chip 2007, 7 (7), 868-875.

(130) Rezayan, A. H.; Mosavi, M.; Kheirjou, S.; Amoabediny, G.; Ardestani, M. S.; Mohammadnejad, J. Monodisperse Magnetite (Fe3O4) Nanoparticles Modified with Water Soluble Polymers for the Diagnosis of Breast Cancer by MRI Method. J. Magn. Magn. Mater. 2016, 420, 210-217.

(131) Mashhadi Malekzadeh, A.; Ramazani, A.; Tabatabaei Rezaei, S. J.; Niknejad, H. Design and Construction of Multifunctional Hyperbranched Polymers Coated Magnetite Nanoparticles for Both Targeting Magnetic Resonance Imaging and Cancer Therapy. J. Colloid Interface Sci. 2017, 490, 64-73.

(132) Aliabadi, M.; Shagholani, H.; Yunessnia lehi, A. Synthesis of a Novel Biocompatible Nanocomposite of Graphene Oxide and Magnetic Nanoparticles for Drug Delivery. Int. J. Biol. Macromol. 2017, 98, 287-291.

(133) Oroujeni, M.; Kaboudin, B.; Xia, W.; Jönsson, P.; Ossipov, D. A. Conjugation of Cyclodextrin to Magnetic Fe3O4 Nanoparticles via
Polydopamine Coating for Drug Delivery. Prog. Org. Coat. 2018, 114, 154-161.

(134) Zhang, X.; Le, T. A.; Yoon, J. Development of a Real Time Imaging-Based Guidance System of Magnetic Nanoparticles for Targeted Drug Delivery. J. Magn. Magn. Mater. 2017, 427, 345-351.

(135) Ansari, M.; Bigham, A.; Hassanzadeh-Tabrizi, S. A.; Abbastabar Ahangar, H. Synthesis and Characterization of $\mathrm{Cu} 0.3 \mathrm{Zn} 0.5 \mathrm{Mg} 0.2-$ Fe2O4 Nanoparticles as a Magnetic Drug Delivery System. J. Magn. Magn. Mater. 2017, 439, 67-75.

(136) Patitsa, M.; Karathanou, K.; Kanaki, Z.; Tzioga, L.; Pippa, N.; Demetzos, C.; Verganelakis, Di. A.; Cournia, Z.; Klinakis, A. Magnetic Nanoparticles Coated with Polyarabic Acid Demonstrate Enhanced Drug Delivery and Imaging Properties for Cancer Theranostic Applications. Sci. Rep. 2017, 7 (1), 775.

(137) Berenguel-Alonso, M.; Granados, X.; Faraudo, J.; AlonsoChamarro, J.; Puyol, M. Magnetic Actuator for the Control and Mixing of Magnetic Bead-Based Reactions on-Chip. Anal. Bioanal. Chem. 2014, 406 (26), 6607-6616.

(138) Kolosnjaj-Tabi, J.; Wilhelm, C. Magnetic Nanoparticles in Cancer Therapy: How Can Thermal Approaches Help? Nanomedicine 2017, 12 (6), 573-575.

(139) Moy, A. J.; Tunnell, J. W. Combinatorial Immunotherapy and Nanoparticle Mediated Hyperthermia. Adv. Drug Delivery Rev. 2017, $114,175-183$. 